\title{
Dendritic cells maintain dermal adipose-derived stromal cells in skin fibrosis
}

\author{
Jennifer J. Chia, ${ }^{1,2,3}$ Tong Zhu, ${ }^{3,4}$ Susan Chyou, ${ }^{3}$ Dragos C. Dasoveanu, ${ }^{3,5}$ Camila Carballo, ${ }^{6}$ Sha Tian, ${ }^{3}$ Cynthia M. Magro, ${ }^{7}$ \\ Scott Rodeo, ${ }^{6,8}$ Robert F. Spiera, ${ }^{9}$ Nancy H. Ruddle, ${ }^{10}$ Timothy E. McGraw, ${ }^{11}$ Jeffrey L. Browning, ${ }^{12}$ Robert Lafyatis, ${ }^{13}$ \\ Jessica K. Gordon, ${ }^{9}$ and Theresa T. Lü,3,9,14,15
}

\begin{abstract}
'Weill Cornell/Rockefeller/Sloan Kettering Tri-Institutional MD-PhD Program, New York, New York, USA. Immunology and Microbial Pathogenesis Program, Weill Cornell Graduate School of Medical Sciences, New York, New York, USA. ${ }^{3}$ Autoimmunity and Inflammation Program, Hospital for Special Surgery, New York, New York, USA. ${ }^{4}$ Xiangya Hospital, Central South University, Changsha, Hunan, China. 5Physiology, Biophysics and Systems Biology Program, Weill Cornell Graduate School of Medical Sciences, New York, New York, USA. ${ }^{6}$ Tissue Engineering, Regeneration, and Repair Program, Hospital for Special Surgery, New York, New York, USA. 'Department of Pathology, Weill Cornell Medical College, New York, New York, USA. ${ }^{8}$ Orthopedic Surgery, Hospital for Special Surgery, New York, New York, USA. ${ }^{9}$ Rheumatology, Hospital for Special Surgery, New York, New York, USA. ${ }^{10}$ Department of Epidemiology of Microbial Diseases, Yale School of Public Health, New Haven, Connecticut, USA. "Department of Biochemistry, Weill Cornell Medical College, New York, New York, USA. ${ }^{2}$ Microbiology Department, Boston University School of Medicine, Boston, Massachusetts, USA. ${ }^{3}$ Division of Rheumatology and Clinical Immunology, University of Pittsburgh Medical Center, Pittsburgh, Pennsylvania, USA. ${ }^{14}$ Pediatric Rheumatology, Hospital for Special Surgery, New York, New York, USA. ${ }^{15}$ Department of Microbiology and Immunology, Weill Cornell Medical College, New York, New York, USA.

Scleroderma is a group of skin-fibrosing diseases for which there are no effective treatments. A feature of the skin fibrosis typical of scleroderma is atrophy of the dermal white adipose tissue (DWAT). Adipose tissue contains adipose-derived mesenchymal stromal cells (ADSCs) that have regenerative and reparative functions; however, whether DWAT atrophy in fibrosis is accompanied by ADSC loss is poorly understood, as are the mechanisms that might maintain ADSC survival in fibrotic skin. Here, we have shown that DWAT ADSC numbers were reduced, likely because of cell death, in 2 murine models of scleroderma skin fibrosis. The remaining ADSCs showed a partial dependence on dendritic cells (DCs) for survival. Lymphotoxin $\beta$ (LT $\beta$ ) expression in DCs maintained ADSC survival in fibrotic skin by activating an LT $\beta$ receptor/ $\beta$ integrin (LT $\beta R / \beta_{1}$ integrin) pathway on ADSCs. Stimulation of LT $\beta R$ augmented the engraftment of therapeutically injected ADSCs, which was associated with reductions in skin fibrosis and improved skin function. These findings provide insight into the effects of skin fibrosis on DWAT ADSCs, identify a DC-ADSC survival axis in fibrotic skin, and suggest an approach for improving mesenchymal stromal cell therapy in scleroderma and other diseases.
\end{abstract}

\section{Introduction}

Scleroderma is a group of skin-fibrosing diseases that includes systemic sclerosis, which is characterized by skin and internal organ fibrosis, and localized scleroderma, which affects mainly the skin $(1,2)$. Scleroderma skin fibrosis is associated with high morbidity. It can be painful, lead to severe joint contractures and loss of hand function, and, in children, cause asymmetric limb or facial growth $(1,2)$. Wound healing is also compromised (1). There are no effective treatments for skin fibrosis, underscoring the need to better understand the biologic processes involved.

One of the features of scleroderma skin fibrosis is the loss of hypodermal adipose tissue, more recently termed "dermal white adipose tissue" (DWAT) (3), which we will use herein. Fleischmajer and colleagues performed excisional biopsies down to the muscle layer and documented DWAT loss and replacement with fibrotic tissue in scleroderma patients (4). DWAT loss is also observed in murine scleroderma models (5-10), which more readily allow evaluation of all skin layers upon sacrifice. DWAT loss involves loss of

Authorship note: T. Zhu and S. Chyou contributed equally to this work Conflict of interest: The authors have declared that no conflict of interest exists. Submitted: November 30, 2015; Accepted: August 30, 2016.

Reference information: J Clin Invest. 2016;126(11):4331-4345. doi:10.1172/JCI85740. adipocytes, and this at least in part reflects a direct contribution of adipocytes to fibrosis, as recent studies have shown that adipocytes can transdifferentiate into myofibroblasts in vitro and in vivo in skin fibrosis $(6,11)$. In addition to adipocytes, adipose tissue is also composed of a stromal-vascular fraction, and whether elements of this fraction are also lost with DWAT atrophy is not known.

Adipose-derived mesenchymal stromal cells (ADSCs) are one element of the stromal-vascular fraction. ADSCs have regenerative potential, likely giving rise to committed precursors that renew and expand adipose tissue, and are also capable of differentiating into other mesenchymal lineages (12-14). They are also reparative and exhibit antiinflammatory and angiogenic properties (15), and in skin, they may contribute to initial stages of wound healing (16). Whether ADSC numbers are reduced with DWAT atrophy in skin fibrosis is not understood, but loss of these regenerative and reparative cells would point to ADSC replenishment as a potential therapeutic goal. At the same time, there is a need to understand the mechanisms that maintain endogenous ADSC survival in fibrotic skin, as this could help in developing ADSC treatment strategies.

In this study, we show that ADSC numbers are reduced in skin fibrosis, likely due to cell death, and delineate a mechanism that regulates the survival of the remaining ADSCs in fibrotic skin. Dendritic cells (DCs) are potent antigen-presenting cells that are best known 


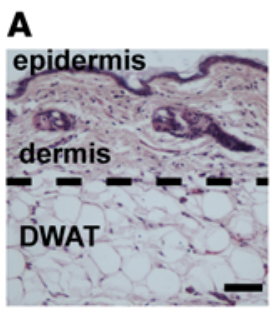

B Unfractionated

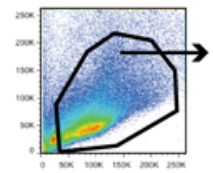
Epidermis/dermis
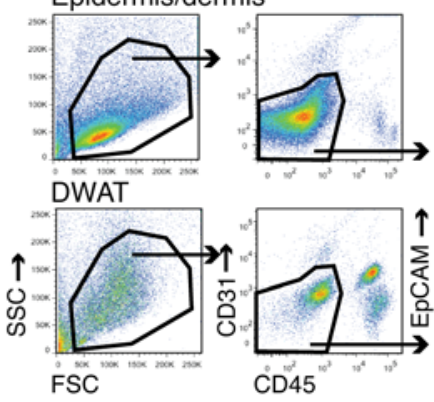

Adipogenic efficiency

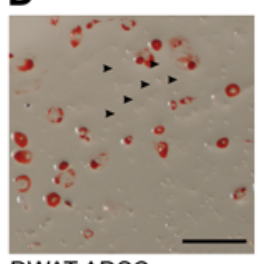

DWAT ADSC

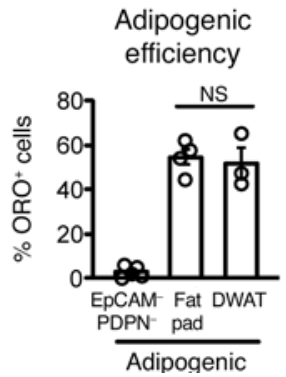

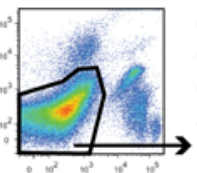
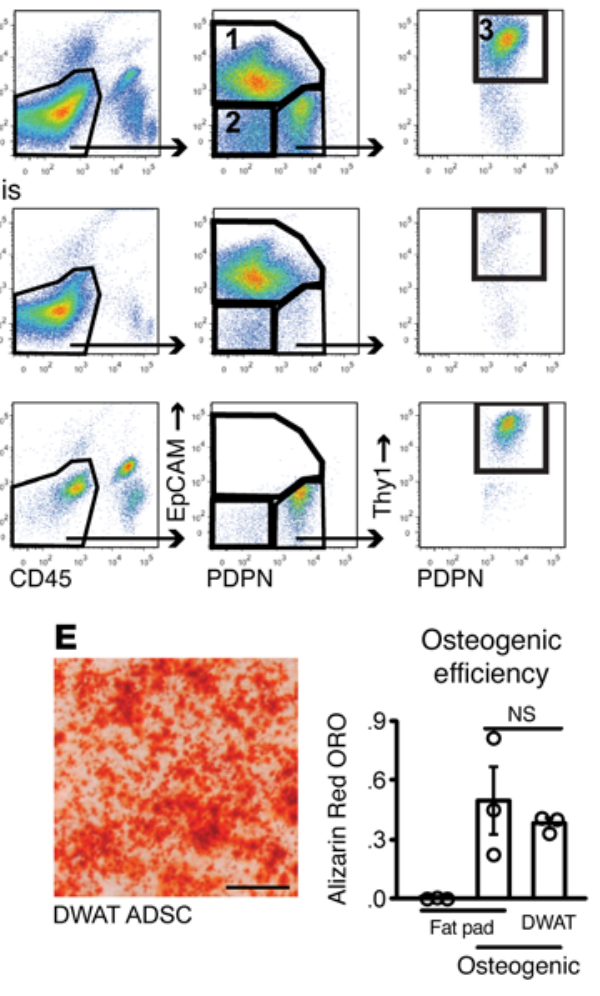

C

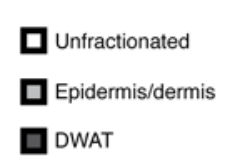

(2) EpCAM $\mathrm{PDPN}^{-}$

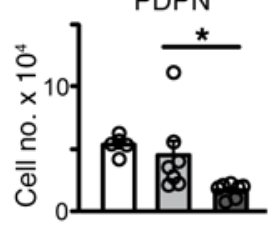

$\mathbf{F}$

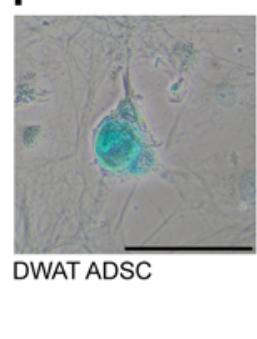

(1) $\mathrm{EpCAM}^{+}$

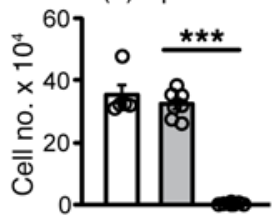

(3) $\mathrm{EpCAM}^{-} \mathrm{PDPN}^{-}$ Thy $1^{+}$

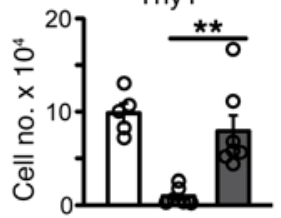

Chondrogenic efficiency

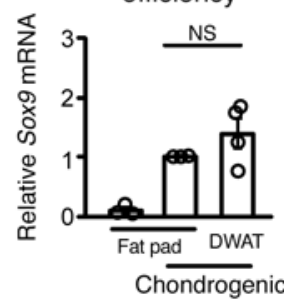

Figure 1. Characterization of DWAT ADSCs. (A) Representative H\&E stain of normal skin. Dashed line indicates the division between the epidermal/ dermal and DWAT fractions. $n=$ at least 3 mice. (B and $\mathbf{C}$ ) Skin was left unfractionated or separated into epidermal/dermal and DWAT fractions and prepared for flow cytometric analysis. $n=5-7$ mice per condition over 3 to 4 experiments. (B) Gating of epidermal and mesenchymal cell populations. (C) Cell numbers of each population in indicated fraction. Numbers are reported per 8-mm punch. (D) Adipocyte differentiation of isolated EpCAM-PDPN- cells, inguinal fat pad ADSCs, or isolated DWAT ADSCs. Left: Representative Oil Red O-stained (ORO-stained) culture. Arrowheads point to undifferentiated cells. Right: Adipogenic efficiency. (E) Osteogenic differentiation of inguinal fat pad ADSCs or isolated DWAT ADSCs. Left: Representative Alizarin Redstained culture. Right: Osteogenic efficiency. (F) Chondrogenic differentiation of inguinal fat pad ADSCs or isolated DWAT ADSCs. Left: Representative chondro-nodule in Alcian blue-stained culture. Right: Chondrogenic efficiency. (D-F) Symbols represent independent experiments. $n=3-5$. Scale bars: 50 $\mu \mathrm{m}$. ${ }^{*} P<0.05,{ }^{* *} P<0.01,{ }^{* *} P<0.001$ using 2-tailed unpaired Student's $t$ test. Error bars depict the SEM.

for their role in adaptive immunity (17), but we have recently shown that DCs also maintain survival of lymph node fibroblastic reticular cells in inflamed lymph nodes (18). We show here that many skin DCs localize to the DWAT, where they maintain ADSC survival in fibrotic skin via lymphotoxin $\beta$ (LT $\beta)$ and stimulation of an LT $\beta$ receptor $/ \beta_{1}$ integrin (LT $\beta \mathrm{R} / \beta_{1}$ integrin) pathway in ADSCs. We further show that stimulation of LT $\beta$ R improves engraftment of injected ADSCs, which is associated with DWAT reconstitution and reduction in dermal fibrosis. These data provide insight into the effects of skin fibrosis on DWAT ADSCs, identify a new DC-ADSC axis that maintains ADSC survival in fibrotic skin, and suggest a strategy to improve existing ADSC and other mesenchymal stromal cell therapies.

\section{Results}

DWAT ADSC numbers are reduced upon fibrosis induction. We used flow cytometry combined with manual separation of the epidermal and dermal layers from the DWAT of the back skin (Figure 1A) to identify and characterize ADSCs. In unfractionated skin, we examined CD31-CD45 nonendothelial, nonhematopoietic cells using antibodies to EpCAM to identify presumed epidermal cells (19), to podoplanin (PDPN), which is expressed by DC-regulated lymph node reticular cells, adipocyte progenitors, and bone mar- row-derived mesenchymal stromal cells $(18,20,21)$, and to Thy1, which is expressed by ADSCs and mesenchymal stromal cells (15). We identified 3 major populations in unfractionated skin: $\mathrm{EpCAM}^{+}$ cells, EpCAM-PDPN ${ }^{-}$cells, and EpCAM ${ }^{-}$PDPN $^{+}$Thy $1^{+}$cells (populations 1, 2, and 3, respectively, in Figure 1B). Upon separation of skin layers into epidermal/dermal and DWAT fractions, EpCAM ${ }^{+}$ cells, as expected, were exclusively in the epidermal/dermal fraction; EpCAM-PDPN ${ }^{-}$cells were enriched in the epidermal/dermal fraction, and EpCAM-PDPN ${ }^{+}$Thy $1^{+}$cells were found mainly in the DWAT (Figure 1, B and C). CD34, Sca1, and $\beta_{1}$ integrin (also called CD29) are expressed by fat pad and skin cells that can become adipocytes (22), and DWAT EpCAM-PDPN ${ }^{+}$Thy $1^{+}$cells expressed these markers at levels similar to those of fat pad EpCAM-PD$\mathrm{PN}^{+} \mathrm{Thy}^{+}$cells (Supplemental Figure 1A; supplemental material available online with this article; doi:10.1172/JCI85740DS1). Functionally, DWAT EpCAM-PDPN ${ }^{+}$Thy $^{+}{ }^{+}$cells differentiated into adipocytes, osteoblasts, and chondrocytes with an efficiency similar to that of well-studied inguinal fat pad ADSCs (Figure 1, D-F, and Supplemental Figure 1B) $(13,14)$. In contrast, EPCAM ${ }^{-}$PDPN $^{-}$ cells were largely CD34-Sca1- (Supplemental Figure 1A) and did not show adipocyte differentiation potential (Figure 1D). Together, our results indicated that DWAT EpCAM-PDPN ${ }^{+} \mathrm{Thy}^{+}$cells 
A

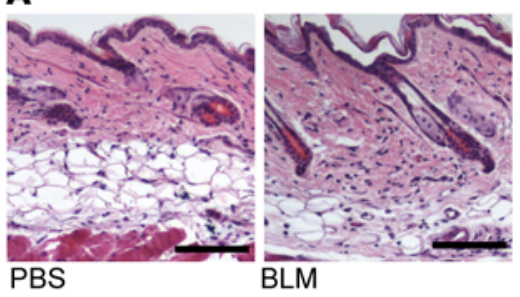

E

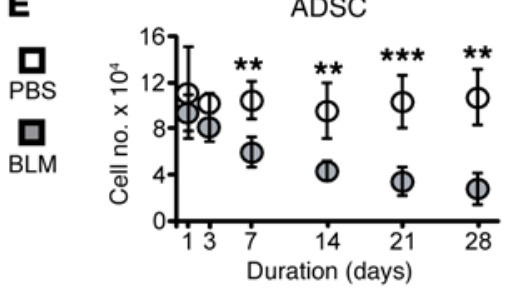

B

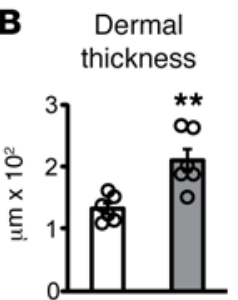

$\mathbf{F}$

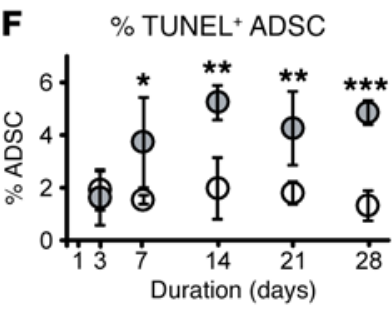

C Collagen

content
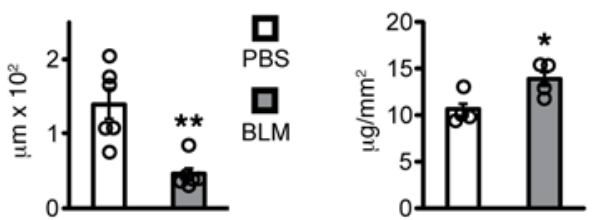

G

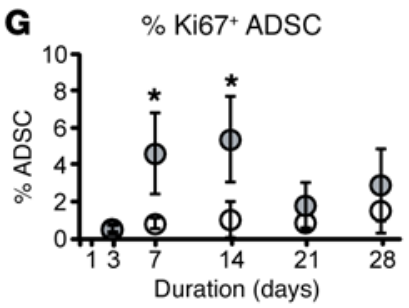

D $\quad T g f b 1$

mRNA
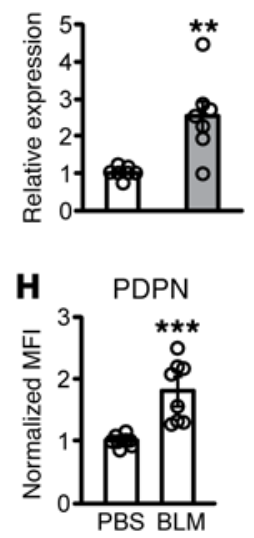

Figure 2. DWAT ADSC numbers are reduced upon fibrosis induction. Mice were injected with PBS or BLM s.c. in back skin over 20 to 28 days unless otherwise indicated. $n=4-8$ mice over 2 to 4 experiments. (A) Representative H\&E stain. (B) Dermal and DWAT thicknesses. (C) Collagen content expressed as micrograms collagen per millimeter of tissue section length. (D) Relative Tgfb1 mRNA. (E-H) ADSCs were assessed by flow cytometry. (E) ADSC numbers per 8-mm punch. (F) Percentage of ADSCs that are TUNEL+. (G) Percentage of ADSCs that are Ki67+. (H) PDPN geometric mean fluorescence intensity (MFI) on ADSCs normalized to PBS group. Scale bars: $100 \mu \mathrm{m} .{ }^{*} P<0.05,{ }^{*} P<0.01,{ }^{* *} P<0.001$ using 2 -tailed unpaired Student's $t$ test. Error bars depict the SD in E-G and the SEM in the other graphs.

represented ADSCs while EpCAM-PDPN ${ }^{-}$cells were composed of non-ADSC mesenchymal cells such as fibroblasts.

We examined ADSC numbers over time in the widely used bleomycin-induced (BLM-induced) skin fibrosis model. This model is thought to be driven by BLM-induced oxidative stress (23), and has a gene expression profile that is consistent with 1 of the 3 major gene expression profiles of systemic and localized scleroderma patients as described by Whitfield and colleagues (24). Histologically, the skin is characterized by increased dermal thickness and DWAT atrophy at BLM injection sites in back skin after 3 weeks (Figure 2, A and B) $(5,23)$. There is also increased collagen deposition and $\mathrm{Tg} f b 1 \mathrm{mRNA}$ (Figure 2, C and D) $(5,23)$. We found that ADSC numbers declined gradually starting at day 7 (Figure 2E), correlating with the start of DWAT loss (Supplemental Figure 1C). As adipocytes differentiate into myofibroblasts during fibrosis $(6,11)$, we asked whether ADSC loss might reflect differentiation into myofibroblasts and assessed myofibroblast numbers. Myofibroblasts are identified by their expression of $\alpha$-smooth muscle actin (SMA) and express Sca1 at intermediate levels in BLM-induced lung fibrosis (25). $\mathrm{SMA}^{+}$cells in homeostatic skin include arteriolar smooth muscle cells and those of the arrector pili muscles, and $\mathrm{SMA}^{+}$cells in control skin were Sca1- (Supplemental Figure 1D). Upon BLM treatment, $\mathrm{SMA}^{+-}$ Sca1 ${ }^{\text {med }}$ presumed myofibroblasts were detected as early as day 3 , and the majority of these cells were PDPN $^{-}$(Supplemental Figure $1, \mathrm{D}$ and $\mathrm{E})$. However, the increase in presumed myofibroblast numbers was about 30 -fold less than the loss of ADSCs (compare Supplemental Figure 1E with Figure 2E), suggesting that while ADSCs could have contributed to myofibroblast accumulation, the majority of ADSC loss was not accounted for by this process. $\mathrm{EpCAM}^{+}$and $\mathrm{EpCAM}^{-} \mathrm{PDPN}^{-}$numbers were unchanged at 21 days (Supplemental Figure 1F), further suggesting that ADSCs did not differentiate in great numbers into additional popula- tions. Instead, the reduction in ADSC numbers correlated with their increased TUNEL staining starting at day 7 and maintained through day 28, suggesting decreased survival (Figure 2, E and F). Together, these data showed that there was a large reduction in ADSC numbers with fibrosis induction and suggested that this was primarily due to disrupted survival.

Fibrosis induction was also associated with a change in the state of ADSCs. ADSC proliferation assessed by Ki67 was transiently increased at days 7 and 14 (Figure 2G), potentially reflecting an injury response and an attempt to compensate for ADSC loss. Additionally, PDPN levels on the remaining ADSCs were increased upon BLM treatment (Figure 2H). PDPN upregulation is found at sites of inflammation associated with fibrosis (26), and in lymph nodes, PDPN upregulation on reticular cells is associated with DC dependence for survival (18). Taken together, these results show that ADSCs were fewer in fibrotic skin, likely because of disrupted survival, and those remaining were in a different, potentially DC-dependent, state.

DCs are localized to the DWAT in homeostatic and fibrotic skin. Murine dermal DCs have been identified and characterized mainly in ear skin, which has no DWAT $(17,27)$, so we asked whether DCs could also be identified in the DWAT. We used the gating strategy of Tamoutounour and colleagues (27), which identifies $\mathrm{CD}_{11 b^{-}}$and $\mathrm{CD} 11 \mathrm{~b}^{+} \mathrm{DC}$ populations as well as monocytes (P1), 2 populations of monocyte-derived DCs (P2 and P3), and $\mathrm{MHCII}^{-}$ and $\mathrm{MHCII}^{+}$macrophages (P4 and $\mathrm{P} 5$, respectively). For clarity, we refer to the gated $\mathrm{CD}_{11 b^{-}}$and $\mathrm{CD}_{11 b^{+}} \mathrm{DC}$ populations as "DCs," regardless of origin, and the P2 and P3 populations as "P2 monocyte-derived DCs" and "P3 monocyte-derived DCs." We identified each of these populations in back skin (Supplemental Figure 2A), and sought to confirm our DC gating. ZBTB46 is a transcription factor that, among mature hematopoietic cells, is specific for nonplasmacytoid DCs $(28,29)$ that, at homeostasis, 


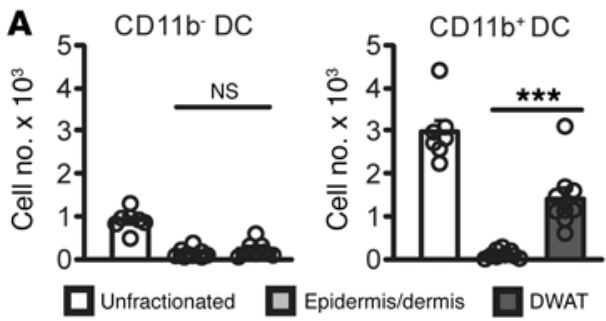

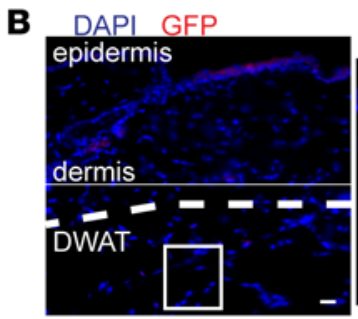

WT
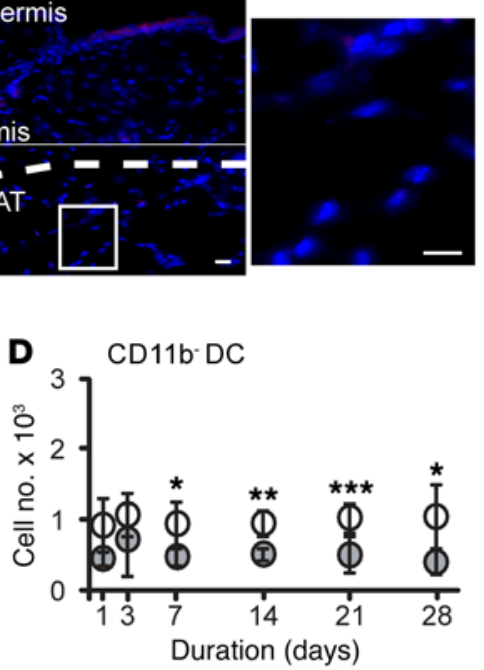

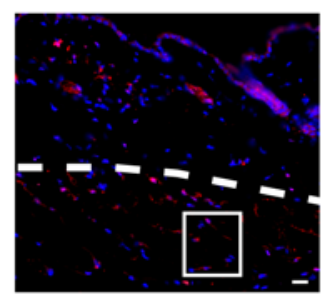

zDCGFP/GFP chimera
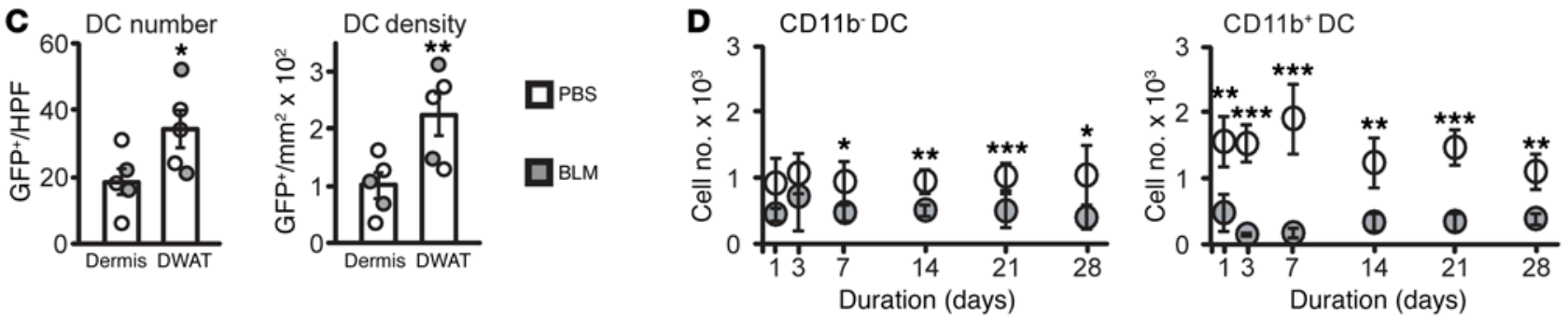
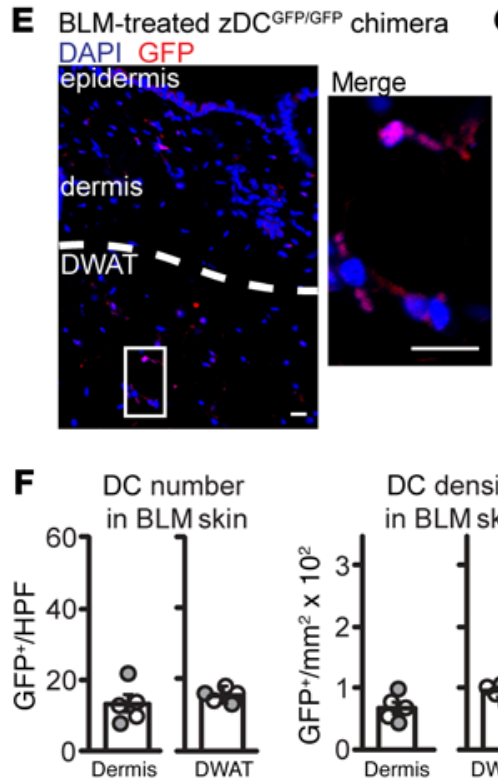

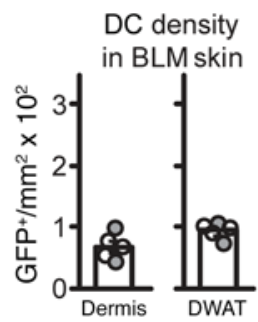

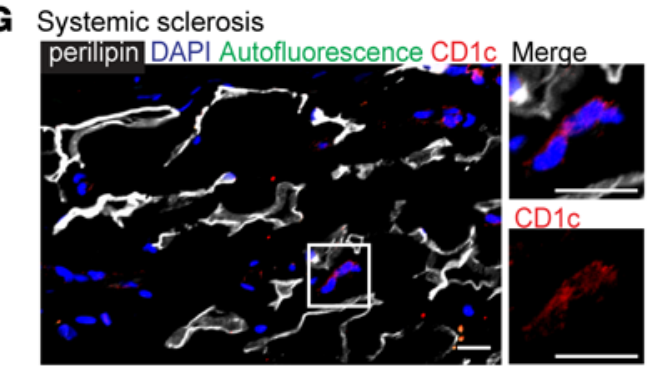
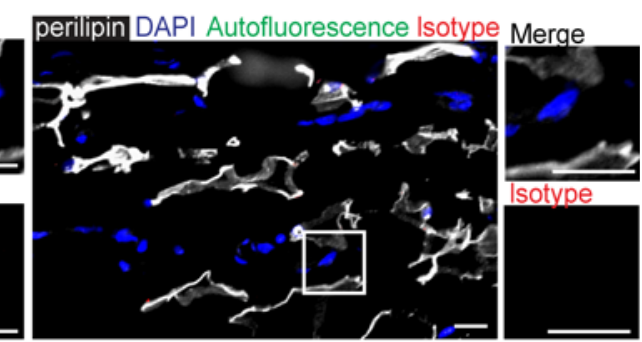

Figure 3. DCs are localized in the DWAT of homeostatic and fibrotic skin. (A) DC numbers in unfractionated skin or indicated skin fraction as assessed by flow cytometry. Numbers are reported per 8-mm punch. $n=5-7$ mice per condition over 3 to 4 experiments. (B and C) Back skin from WT mice or zDC ${ }^{\text {CFP/ }}$ CFP $\rightarrow$ WT chimeras (ZDC CFP/GFP chimera) was stained for GFP (red) and DAPI (blue). Representative of $n=2$ mice per condition. (B) Representative images. Dashed line indicates division between dermis and DWAT. (C) GFP+ cell numbers (left) and GFP+ cells per area (right) in the dermis and DWAT of ZDCCFP/GFP chimeras. Each symbol represents a field; similarly shaded symbols are from the same chimera. (D) DC numbers per punch as assessed by flow cytometry in WT mice treated with PBS or BLM as indicated. $n=4-8$ over 2 to 4 experiments. (E and F) ZDCCFP/CFP chimeras were treated with BLM for 21 days, and then back skin was stained for GFP (red) and DAPI (blue). $n=2$ chimeras. (E) Representative image. Dashed line indicates division between dermis and DWAT. (F) GFP+ cell numbers (left) and GFP+ cells per area (right). Each symbol represents a field; similarly shaded symbols are from the same chimera. HPF high-power field. (G and $\mathbf{H}$ ) Human systemic sclerosis (SSc) lesional skin sections stained for CD1c (red), perilipin (white), and DAPI (blue). $n=3$ individuals. (C) Representative images. Orange particles are autofluorescent and nonspecific. Insets highlight nucleated CD1c ${ }^{+}$cells. (H) CD1c ${ }^{+}$cells per area. Each symbol represents a field; similarly shaded symbols are from the same patient. Scale bars: $20 \mu \mathrm{m}$. ${ }^{*} P<0.05,{ }^{* *} P<0.01,{ }^{* * *} P<0.001$ using 2-tailed unpaired Student's $t$ test. Error bars depict the SD in $\mathbf{D}$ and the SEM in other graphs.

develop from common DC progenitors in an FLT3 ligand-dependent manner and, during inflammation, may also develop from monocytes $(28,29)$. We confirmed the identity of the CD11b and CD $11 b^{+}$DCs by their GFP expression in Zbtb46 ${ }^{\text {GFP/GFP }}$ reporter mice (hereafter referred to as $\mathrm{zDC}^{\mathrm{GFP} / \mathrm{GFP}}$ mice) (28), noting that other populations delineated by Tamoutounour and colleagues, including the P2 monocyte-derived DCs and P3 monocyte-derived DCs, did not express ZBTB46 (Supplemental Figure 2, A and B). The identities of $\mathrm{CD}_{11 \mathrm{~b}}$ and $\mathrm{CD} 11 \mathrm{~b}^{+}$DCs were further confirmed by their dependence on FLT3 ligand (17) (Supplemental Figure 2C). We fractionated the skin, finding that this procedure resulted in some DC loss, as the DC numbers from epidermal/dermal and DWAT fractions combined were less than the total in unfractionated skin (Figure 3A). Although this loss made it difficult to evaluate absolute numbers, we could observe that CD11b- DCs were found equally in both the epidermal/dermal and DWAT fractions, 
A

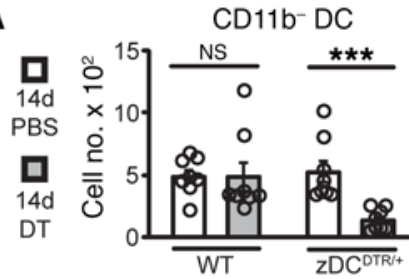

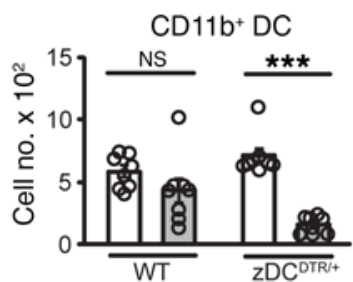

B

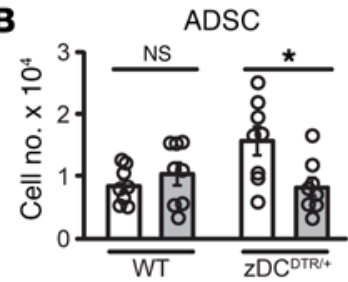

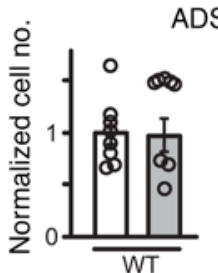

ADSC

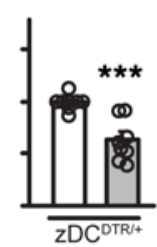

C

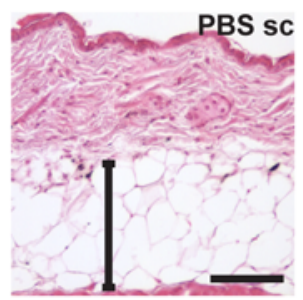

E

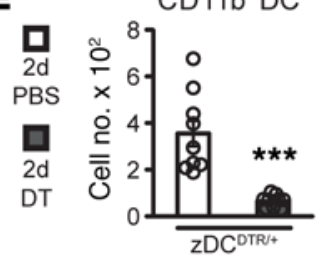

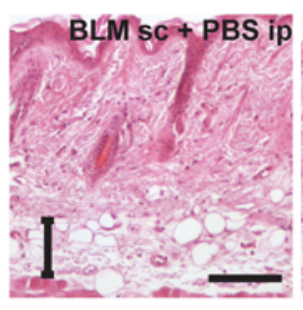

CD11b+DC
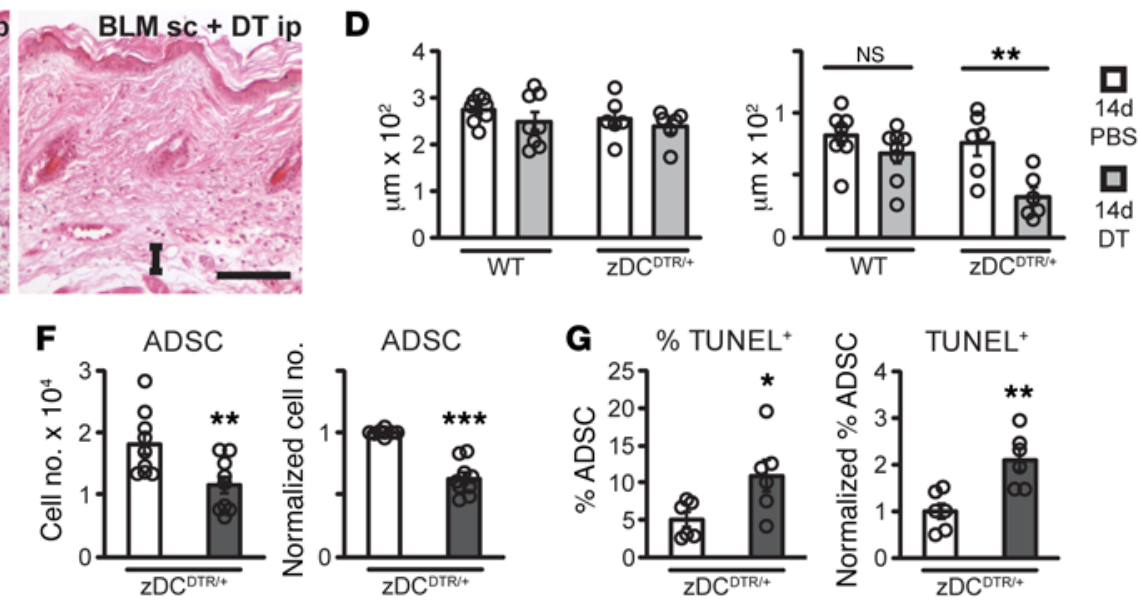

H

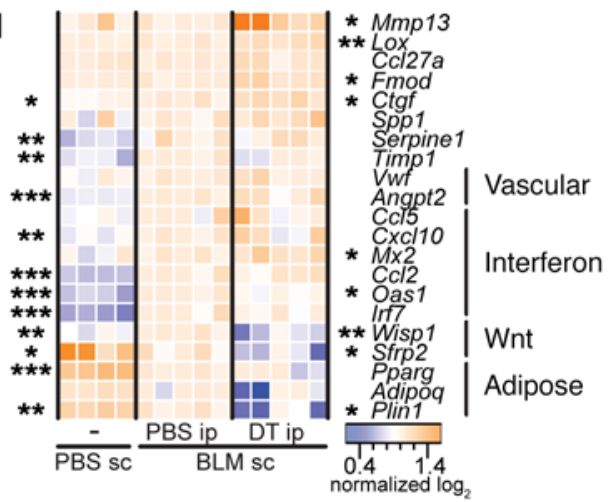

\section{I}

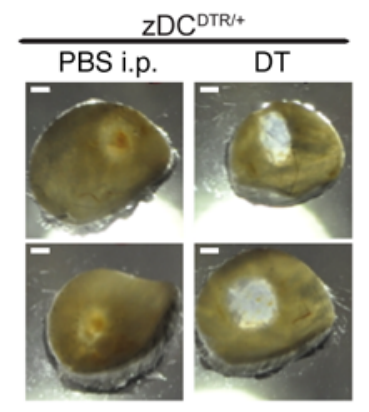

J

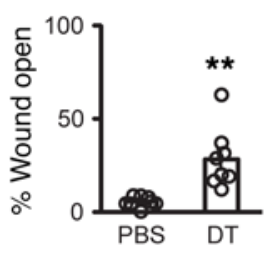

Figure 4. DCs maintain ADSC survival and skin function in BLM-induced fibrosis. (A-D) WT $\rightarrow$ WT chimeras $(W T)$ or $\mathrm{ZDC}^{\mathrm{DTR} /+} \rightarrow$ WT chimeras $\left(z D C C^{\mathrm{DTR} /+}\right)$ were treated s.c. with BLM over 35 days, with i.p. PBS or DT over the final 14 days before skin analysis. (A) DC numbers per punch. (B) ADSC numbers. Left: absolute numbers per punch; right: normalized to PBS group. (C) Representative H\&E-stained sections. For comparison, left panel shows a zDCDTR/+ chimera treated with PBS s.c. for 35 days. Scale bars: $100 \mu \mathrm{m}$. (D) Dermal and DWAT thicknesses. (E-G) zDC ${ }^{\mathrm{DTR} /+}$ chimeras were treated with BLM over 22 days, with PBS or DT for the final 2 days before analysis. (E) DC numbers per punch. (F) ADSC numbers. Left: absolute numbers per punch; right: normalized. (G) TUNEL ${ }^{+}$ADSCs. Left: percentage of ADSCs that are TUNEL ${ }^{+}$; right: normalized. (A-G) $n=6-8$ chimeras per condition over 3-6 experiments. (H) $\mathrm{zDC} \mathrm{CTR} /+^{+}$chimeras were treated as in A-D. Relative mRNA expression of indicated genes using NanoString or, for Adipoq, quantitative PCR for first 2 BLM samples. Each column represents 1 mouse; $n=4-5$ per condition over 3 experiments. Statistical significance of differences between the PBS s.c. and the BLM s.c. plus PBS i.p. groups is shown at left, and of differences between the BLM s.c. plus PBS i.p. and the BLM s.c. plus DT i.p. groups at right. (I and J) $\mathrm{zDC}^{\mathrm{DTR} /+}$ chimeras were treated with BLM for 22 days, receiving PBS i.p. or DT i.p. from day 20 onward. Full-thickness wounds were inflicted the day after BLM cessation and analysis performed 14 days later (see Supplemental Figure 4R). $n=8$ wounds in 4 mice per condition over 2 experiments. (I) Representative wounds. Scale bars: $1 \mathrm{~mm}$. (J) Percentage open at day 14 relative to wound size at day $0 .{ }^{*} P<0.05,{ }^{* *} P<0.01,{ }^{* *} P<0.001$ using 2 -tailed unpaired Student's $t$ test. Error bars depict the SEM.

while CD11b+ DCs were primarily in the DWAT, where they were enriched relative to the CD11b- DC population (Figure 3A).

We confirmed our flow cytometric findings on tissue sections using $\mathrm{zDC}^{\mathrm{GFP} / \mathrm{GFP}}$ reporter mice. Because ZBTB46 is also expressed by endothelial cells, we reconstituted lethally irradiated WT hosts with bone marrow from $\mathrm{zDC}^{\mathrm{GFP} / \mathrm{GFP}}$ mice to generate $\mathrm{zDC}^{\mathrm{GFP} / \mathrm{GFP}} \rightarrow \mathrm{WT}$ chimeras with $\mathrm{GFP}^{+}$DCs and WT endothelial cells (28). DCs in tissue sections of the $\mathrm{zDC}^{\mathrm{GFP} / \mathrm{GFP}} \rightarrow \mathrm{WT}$ chimeras were more numerous and at higher density in the DWAT than dermis (Figure 3, B and C). Our results together showed that, at homeostasis in back skin, DCs were located in both the dermis and DWAT and the majority of DWAT DCs were CD11b+.

We next characterized DCs during fibrosis induction. Upon BLM treatment, cells gated as CD11b and CD11b ${ }^{+}$DCs expressed GFP in $\mathrm{zDC}^{\mathrm{GFP} / \mathrm{GFP}}$ mice and remained the only populations to do so (Supplemental Figure 3A), consistent with our findings in homeo- 
static skin. CD11b- DC numbers were reduced by $49 \%$ from day 7 after BLM initiation onward, while CD11b+ DC numbers were affected more dramatically and immediately, with a $69 \%$ reduction by day 1 (Figure 3D). DC numbers were not increased in draining lymph nodes (Supplemental Figure 3B), and the remaining CD11 ${ }^{+}$ DCs had increased TUNEL (Supplemental Figure 3C), indicating that DC loss was due to cell death rather than to migration from the skin to draining lymph nodes. We further observed a reduction in Flt3 ligand mRNA and protein in fibrotic skin (Supplemental Figure 3, D and E), suggesting the possibility that loss or dysfunction of FLT3 ligand-expressing cells may have contributed to sustained DC loss. Together, our results suggest that many of the DCs die by 1 day of BLM treatment, likely reflecting direct effects of BLM.

We also enumerated the other mononuclear phagocyte populations upon BLM treatment. Monocytes and P2 monocytederived DCs showed an early and transient increase that is consistent with the findings of others in the $\operatorname{BLM}$ model $(5,30)$, while the combined populations of $\mathrm{P} 3$ monocyte-derived $\mathrm{DCs}$ and $\mathrm{MHCII}^{-}$ and $\mathrm{MHCII}^{+}$macrophages (P3-5) were not increased (Supplemental Figure 3F). Our results together show that DCs are rapidly reduced in the skin upon BLM administration, but that this does not represent a generalized loss of hematopoietic cells, as other mononuclear phagocytes are transiently increased or unchanged.

We asked whether DCs in fibrotic skin remained in the atrophied DWAT. DC loss was greater in the DWAT than in the dermis in BLM-treated $\mathrm{zDC}^{\mathrm{GFP} / \mathrm{GFP}} \rightarrow \mathrm{WT}$ chimeras (compare Figure 3, C and F), but DCs were still present in the DWAT (Figure 3, E and F). We also examined human scleroderma skin, initially staining for $\mathrm{CD}_{11} \mathrm{c}^{+}$cells. However, CD11c levels are 10 times lower on murine DWAT DCs than on epidermal/dermal DCs (data not shown), and we had difficulty detecting CD11c in human DWAT (data not shown). We thus stained for CD1c, a marker of human CD11b ${ }^{+}$DCs $(17,31)$, and assessed DC density. Note that absolute numbers were not assessed because the incomplete DWAT obtained with standard full-thickness punch biopsies precluded this analysis. CD1 ${ }^{+}$ cells were at higher density in the DWAT than in the dermis (Figure 3, G and H). Localization of DCs in the DWAT of murine and human fibrotic skin supported the idea that DCs could potentially regulate ADSCs in fibrosis.

DCs maintain ADSC survival in BLM-induced fibrosis. We asked whether DCs could regulate the survival of the ADSCs that remained in fibrotic skin. We reconstituted lethally irradiated WT hosts with bone marrow from mice transgenic for $Z b t b 46$-driven diphtheria toxin receptor $\left(\mathrm{zDC}^{\mathrm{DTR} /+}\right)(29)$. The resulting $\mathrm{zDC}^{\mathrm{DTR} /+} \rightarrow \mathrm{WT}$ chimeras allowed for radiosensitive $\mathrm{ZBTB}^{+} 6^{+}$cell depletion upon diphtheria toxin (DT) injection. As ZBTB46 was specifically expressed by DCs and not by other mononuclear phagocyte populations in skin (Supplemental Figure 2B and Supplemental Figure 3A), DT injection would specifically deplete DCs in these chimeras. WT $\rightarrow$ WT chimeras served as controls. The chimeras received BLM for 35 days, with PBS or DT in the final 14 days (Supplemental Figure 4A). DC depletion led to a $36 \%$ decrease in ADSC numbers (Figure 4, A and B) and a further loss of remaining DWAT (Figure 4, $\mathrm{C}$ and D). ADSC loss was specific; $\mathrm{EpCAM}^{+}$or $\mathrm{EpCAM}^{-} \mathrm{PDPN}^{-}$cell numbers remained unchanged (Supplemental Figure 4, B and C). DC depletion for 2 days also reduced ADSC numbers (Figure 4, E and F), with remaining ADSCs having increased TUNEL (Figure
$4 G)$. ADSC proliferation, adipocyte numbers, and $\mathrm{SMA}^{+} \mathrm{Sca} 1^{+}$myofibroblast numbers were unchanged (Supplemental Figure 4, D-F). These results suggested that DCs contributed to the maintenance of ADSC survival in fibrotic skin.

DC depletion had no impact on circulating monocytes, skin plasmacytoid DCs, or skin monocytes (Supplemental Figure 4, G-I). However, P2 monocyte-derived DCs and the composite P3-5 population were reduced by about $50 \%$ (Supplemental Figure 4, J and $\mathrm{K}$ ), raising the possibility that DCs maintain ADSCs indirectly by maintaining these other mononuclear phagocyte populations.

Subcutaneous injection of BLM can have systemic effects (23), and DC depletion also led to a trend toward decreasing ADSC numbers and subcutaneous inguinal fat pad mass (Supplemental Figure 4, L and M). DC-depleted chimeras also had lower body weight (Supplemental Figure $4 \mathrm{~N}$ ), potentially reflecting systemic loss of fat mass. Thus, DCs may also maintain ADSCs in subcutaneous depots in BLM-treated mice.

We assessed whether DC depletion in fibrotic skin over 14 days altered additional facets of skin fibrosis. BLM-induced fibrosis was associated with altered expression of select fibrosis- and adipose-associated transcripts $(5,24,32-41)$ (Figure $4 \mathrm{H})$, and DC depletion exacerbated some changes while reversing others and also inducing new changes (Figure 4H). Notably, DC depletion worsened some profibrotic gene changes, such as the upregulation of the TGF- $\beta$-regulated profibrotic cytokine connective tissue growth factor $(C t g f)(24,33)$ and the downregulation of $S f r p 2$, an inhibitor of profibrotic Wnt signaling $(8,40)$ (Figure $4 \mathrm{H})$. However, DC depletion also reversed the upregulation of Wisp1, a profibrotic Wnt target gene (42), and upregulated $M m p 13$, which is increased in pathologic fibrosis but plays a protective role (43) (Figure 4H). Consistent with the further loss of DWAT, DC depletion downregulated the adipocyte lipid droplet-associated protein perilipin (44) (Figure $4 \mathrm{H})$. Thus, DC depletion did not uniformly worsen gene expression associated with BLM-induced fibrosis, but the changes suggested an altered skin state, perhaps reflecting further overall skin injury. Consistent with this idea, skin collagen content was unchanged upon DC depletion (Supplemental Figure 4O), but wound closure, an indicator of skin state and function that is reduced by BLM treatment (Supplemental Figure 4, P and Q), was further reduced by DC depletion (Supplemental Figure 4R and Figure 4, I and J). Together, these results suggested that DCs were important for maintaining ADSC survival and skin function in fibrosis.

$D C$-derived LT $\beta$ maintains ADSC survival in BLM-induced fibrosis. We next asked how DCs maintained ADSC survival in fibrosis. We recently demonstrated that lymph node reticular cell survival in inflamed lymph nodes is mediated by lymphotoxin $\beta$ receptor (LT $\beta R$ ), a member of the TNF receptor superfamily (18). LT $\beta$ R ligands are membrane-bound LT $\alpha_{1} \beta_{2}$ and LIGHT (45), and DC-derived LT $\alpha_{1} \beta_{2}$ has a dominant effect on reticular cell survival (18). Surface $\operatorname{LT} \alpha_{1} \beta_{2}$ is sensitive to collagenase digestion (data not shown), so we stained for intracellular LT $\beta$ and detected it in CD11b ${ }^{+}$DCs but not in CD11b- DCs from BLM-treated skin (Figure $5 A)$. Greater expression of LT $\beta$ by skin CD $11 b^{+}$DCs than by CD11b DCs was consistent with mRNA expression patterns reported for lymphoid tissue DCs (Immunological Genome Project, www. immgen.org) (46). These results raised the possibility that DCs, most likely CD11b+ DCs, could mediate ADSC survival via LT $\beta$. 
A

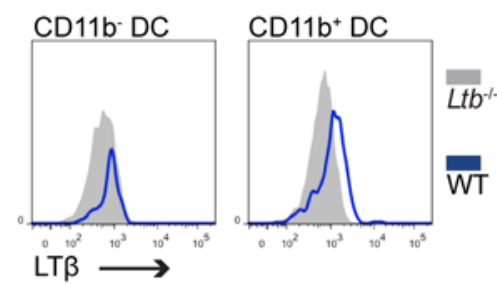

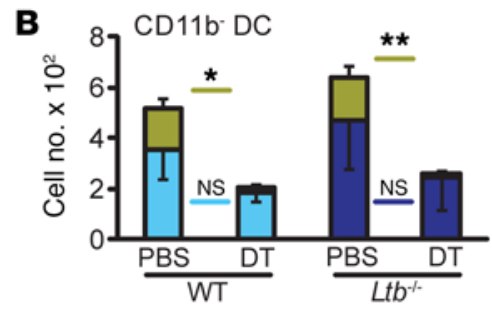

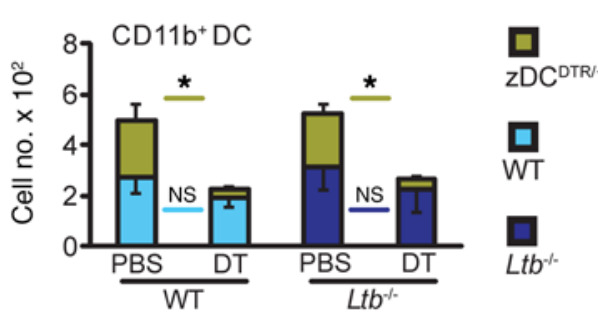

C $\quad \mathrm{ADSC}$
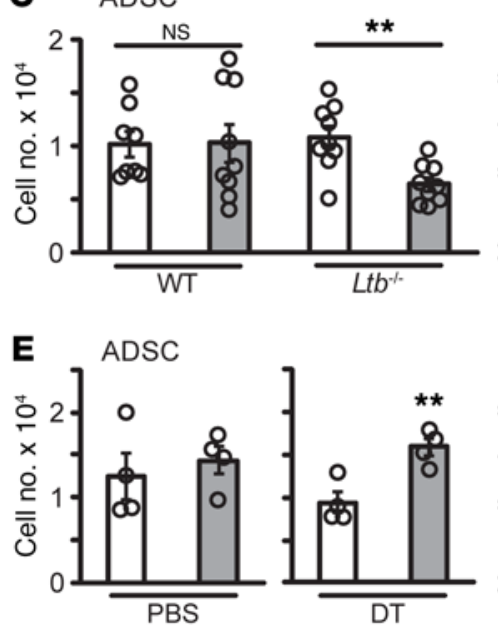

G

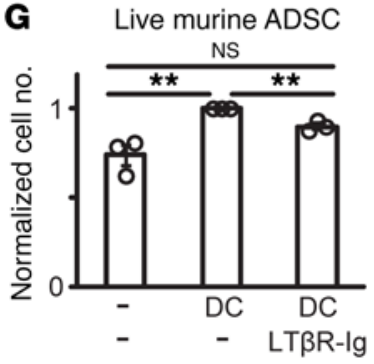

D $\%$ TUNEL+

ADSC
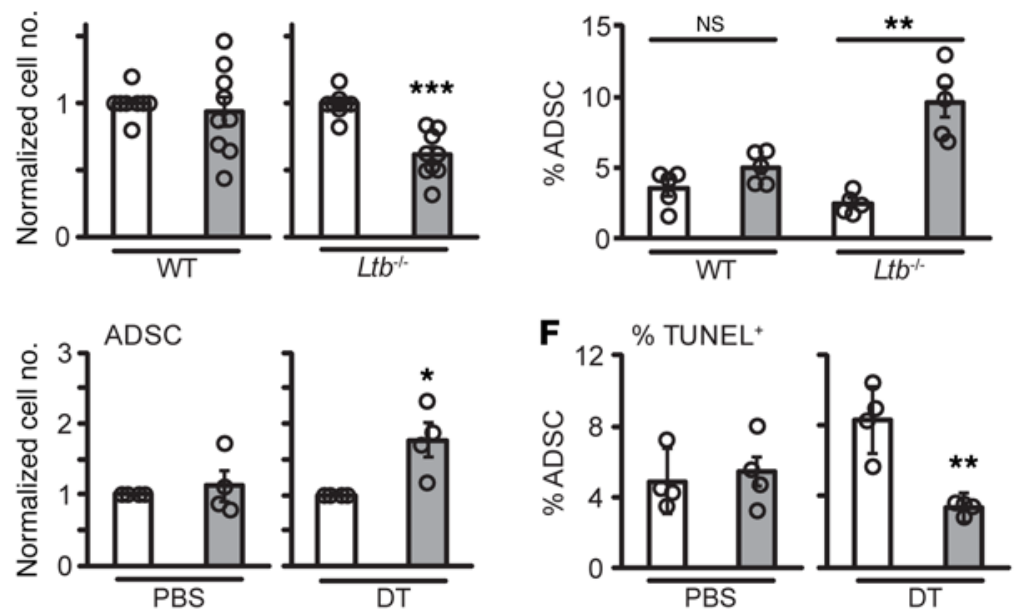

I Live human ADSC

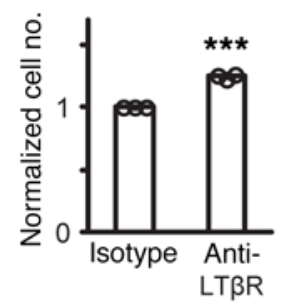

Figure 5. DC-derived LT $\beta$ maintains ADSC survival in BLM-induced fibrosis. (A) Representative histograms of LT $\beta$ staining in skin CD11b- DCs (left) and $\mathrm{CD}_{11 b^{+}} \mathrm{DCs}$ (right) from mice treated with BLM for 21-25 days. $n=6$ WT mice and $3 \mathrm{Ltb}^{-/-}$mice over 3 experiments. (B-D) $z D C^{\mathrm{DTR} /+} / \mathrm{WT} \rightarrow \mathrm{WT}$ mixed chimeras $(\mathrm{WT})$ or $\mathrm{ZDC} \mathrm{DTR} /+^{\mathrm{D}} / \mathrm{Ltb}^{-/-} \rightarrow \mathrm{WT}$ mixed chimeras $\left(L t b^{-/-}\right.$) were injected with BLM over 21 to 22 days, with PBS or DT for the final 1-2 days before skin analysis. (B) DC numbers enumerated by bone marrow donor genotype. Numbers are reported per 8-mm punch. $n=7$ chimeras over 5 experiments. (C) ADSC numbers. Left: absolute numbers per punch; right: normalized to PBS groups. $n=8-9$ chimeras per condition over 7 experiments. (D) Percentage of ADSCs that are TUNEL $+n=5$ chimeras per condition over 5 experiments. (E and $\mathbf{F}$ ) $\mathrm{ZDC}^{\mathrm{DTR} /+}$ chimeras were treated with BLM over 22 days, with PBS or DT for the final 2 days, and with $20 \mu \mathrm{g}$ anti-LT $\beta$ R or isotype control 6 hours before the first dose of PBS or DT. $n=4$ chimeras per condition over 4 experiments. PBS and DT experiments were performed separately using different batches of chimeras. (E) ADSC numbers. Left: absolute numbers per punch; right: normalized. (F) Percentage of ADSCs that are TUNEL+ ( $(\mathbf{G}$ and $\mathbf{H}$ ) CD31-CD45-PDPN+DAPI- cell counts from murine ADSC cultures that were serum-starved and treated with the indicated cells and reagents. (G) Effect on ADSC survival of DCs without or with LT $\beta R$-lg for 48 hours. (H) Effect on ADSC survival of isotype control or agonist anti-LT $\beta R$ for 48 hours. (I) CD31-CD45-PDPN+DAPI- cell counts from human primary ADSC cultures that were serum-starved and treated with isotype control or agonist anti-LT $\beta R$ for 48 hours. (G-I) Each symbol represents 1 of 3 to 5 experiments with 1 to 3 replicate wells per experiment. ${ }^{*} P<0.05$, ${ }^{* *} P<0.01,{ }^{* *} P<0.001$ using 2-tailed unpaired Student's $t$ test. Error bars depict the SEM.

We investigated the importance of DC-derived $\mathrm{LT} \alpha_{1} \beta_{2}$ by generating mixed chimeras in which lethally irradiated WT recipients received $50 \% \mathrm{zDC} \mathrm{zTR} /+^{\mathrm{T}}$ and $50 \% \mathrm{Ltb}^{-/-} \mathrm{Rag}^{-/-}$bone marrow (" $\mathrm{L} t \mathrm{~b}^{-/-}$ mixed chimeras") or, for controls, $50 \% \mathrm{zDC}^{\mathrm{DTR} /+}$ and $50 \%$ WT (i.e., LT $\beta$-sufficient) bone marrow ("WT mixed chimeras"). These chimeras were treated with BLM, and then with control PBS or with DT. Depletion of the DTR ${ }^{+}$DCs left DCs that were LT $\beta$-deficient in the $\mathrm{Ltb}^{-/}$mixed chimeras and DCs that were LT $\beta$-sufficient in the WT mixed chimeras (Supplemental Figure 5A and Figure 5B). This induced DC-specific LT $\beta$ deficiency resulted in a $41 \%$ decrease in ADSCs (Figure 5C), while remaining ADSCs had increased TUNEL (Figure 5D). These results suggested that DCs maintained ADSC survival in fibrotic skin via $\mathrm{LT} \alpha_{1} \beta_{2}$.

Unlike in the $100 \% \mathrm{zDC}^{\mathrm{DTR} /+}$ chimeras (Supplemental Figure 4 , $\mathrm{J}$ and $\mathrm{K}$ ), DC depletion in the mixed chimeras did not lead to a secondary loss of $\mathrm{P} 2$ or $\mathrm{P} 3-5$ populations (Supplemental Figure $5 B)$. This suggested that DC maintenance of ADSC survival was not mediated via P2 or P3-5 mononuclear phagocytes.

We asked whether LT $\beta$ R stimulation was sufficient to prevent DC depletion-induced ADSC loss. We repeated our DC depletion 
A

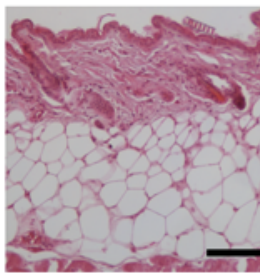

Control

C

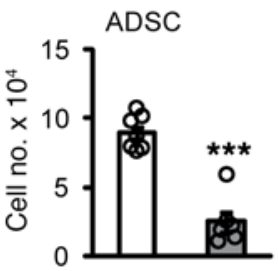

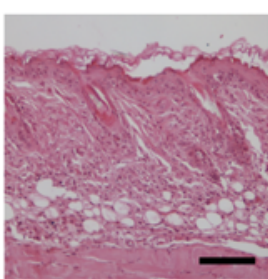

GVHD

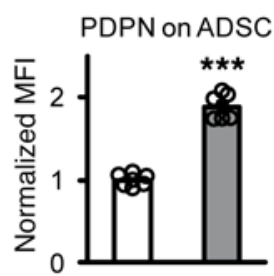

B

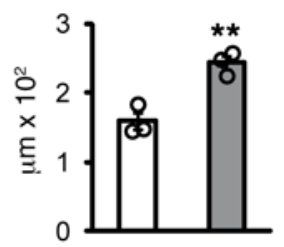

D

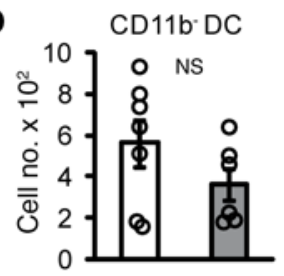

DWAT thickness
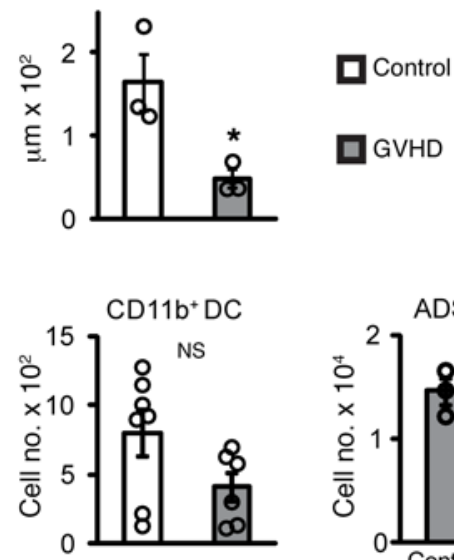

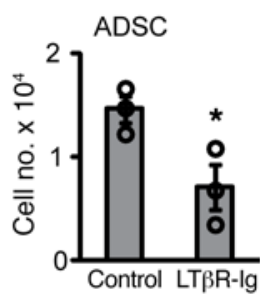

Figure 6. LT $\beta R$ signaling maintains ADSCs in the systemic sclerosis-GVHD model of skin fibrosis. Congenic (control) or B10.D2 (CVHD) splenocytes were adoptively transferred into BALB/c Rag2 ${ }^{-1-}$ mice 22-23 days before sacrifice. Back skin was analyzed. (A) Representative H\&E-stained sections. Scale bars: $100 \mu \mathrm{m}$. (B) Dermal and DWAT thicknesses. (A and B) $n=3$ mice per condition over 2 experiments. (C) ADSC numbers per 8-mm punch. (D) Geometric mean fluorescence intensity (MFI) of PDPN on ADSCs. (E) DC numbers per punch. (C-E) $n=6-7$ mice per condition over 4 experiments. (F) Effect of LT $\beta R$-Ig on ADSC numbers per punch in systemic sclerosis-CVHD mice. Control or LT $\beta R$ - Ig $(100 \mu \mathrm{g})$ was given on day 20 after GVHD induction, and animals were sacrificed on day 22. $n=3$ mice per condition over 2 experiments. ${ }^{*} P<0.05$, ${ }^{*} P<0.01,{ }^{* * *} P<0.001$ using 2 -tailed unpaired Student's $t$ test. Error bars depict the SEM.

studies in BLM-treated 100\% $\mathrm{zDC}^{\mathrm{DTR} /+}$ chimeras as in Figure 4, $\mathrm{E}-\mathrm{G}$, this time administering isotype control or agonist anti-LT $\beta \mathrm{R}$ antibody (47) before the first PBS or DT injection. The anti-LT $\beta R$ was sufficient to upregulate ICAM-1 (Supplemental Figure 5C), known to be downstream of LT $\beta R$ signaling (45). Anti-LT $\beta$ R treatment prevented DC depletion-induced ADSC loss and TUNEL increase (Figure 5, E and F) without affecting ADSC proliferation (Supplemental Figure 5D), suggesting that LT $\beta$ R agonism was sufficient to maintain ADSC survival in the setting of DC depletion.

LT $\beta$ R agonism did not rescue P2 or P3-5 cell numbers (Supplemental Figure 5E), further supporting the idea that these populations were not necessary for DC-mediated maintenance of ADSC survival in fibrotic skin. LT $\beta$ R agonism also did not affect ADSC numbers when DCs were not depleted (Figure 5E). These results further supported the idea that DC-derived LT $\beta$ R signals maintained ADSC survival in fibrotic skin and that this DC-mediated survival mechanism was not mediated by other mononuclear phagocyte populations.

To determine whether DCs or their signals maintained ADSC survival directly, we performed coculture experiments with primary ADSCs and DCs. We used ADSCs from inguinal fat pad as they were numerous, resembled DWAT ADSCs phenotypically and functionally (Figure 1, D-F, and Supplemental Figure 1, A and B), and had also shown dependence on DCs (Supplemental Figure $4 \mathrm{~L}$ ). We sorted CD11b ${ }^{+}$DCs, which expressed LT $\beta$ (Figure 5A), from inguinal fat pad and DWAT together as tissue sources to maximize our yield. Addition of DCs to serum-starved ADSCs increased ADSC survival, and this was inhibited with LT $\beta$ R-Ig, which sequesters LT $\beta R$ ligands (48) (Figure 5G). Furthermore, mimicking the provision of DC-derived $L T \alpha_{1} \beta_{2}$ with agonist anti-LT $\beta R$ at a dose that upregulated ICAM-1 (Supplemental Figure 5F) was sufficient to increase survival of ADSCs in starvation medium (Figure $5 \mathrm{H}$ ). Similarly, agonist anti-human LT $\beta$ R (49) increased the survival of serum-starved human ADSCs (Figure 5I). Together these results suggested that DCs directly maintained ADSC survival via stimulation of LT $\beta R$ on ADSCs.

DCs are not necessary for ADSC maintenance in homeostatic skin, and DC loss does not contribute to ADSC loss during fibrosis induction. Our results showing that DCs maintain ADSC survival in fibrotic skin led us to examine whether DCs or LT $\beta$ R signaling regulated ADSC survival at homeostasis, and whether the BLMinduced loss of $69 \%$ of $\mathrm{CD}_{11} \mathrm{~b}^{+} \mathrm{DCs}$ by day 1 contributed to the subsequent reduction in ADSC numbers during fibrosis induction. At homeostasis, ADSC numbers were unchanged by DC depletion (Supplemental Figure 6, A and B), FLT3 ligand deficiency, or LT $\beta$ deficiency (Supplemental Figure 6C), indicating that DCs do not regulate ADSC survival in the steady state. With fibrosis induction, anti-LT $\beta$ R treatment starting from the initiation of BLM was sufficient to upregulate ICAM-1 but could not prevent ADSC loss at day 7 (Supplemental Figure 6, D-F). These data suggested that ADSC survival at homeostasis was dependent on factors other than DCs and their LT $\beta$ R signals, and that ADSC loss upon fibrosis induction was not attributable to the preceding loss of DCs. Instead, the correlation between ADSC loss and DWAT atrophy suggested that ADSC death was more likely due to loss of critical homeostatic survival factors in the DWAT niche.

LT $\beta R$ signals maintain ADSC numbers in the GHVD model of scleroderma. To test the generality of our findings in the BLM model, we examined the graft-versus-host disease (GVHD) model of systemic sclerosis, a commonly used model that involves the

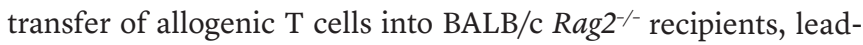
ing to diffuse fibrosis by 3 weeks $(7,50)$. This model is considered to be more reflective of an adaptive immune process, with $\mathrm{T}$ cell activation and development of scleroderma-associated autoantibodies, and also shares gene expression patterns with scleroderma patients $(7,23,24,50)$. Like BLM-treated mice, GVHD mice had dermal thickening, reduced DWAT and ADSC numbers, and elevated ADSC PDPN levels (Figure 6, A-D). We observed 
A

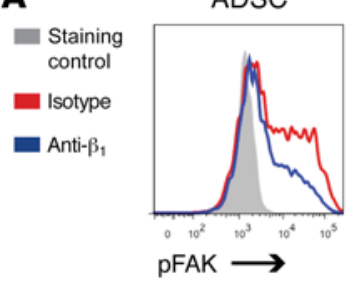

C

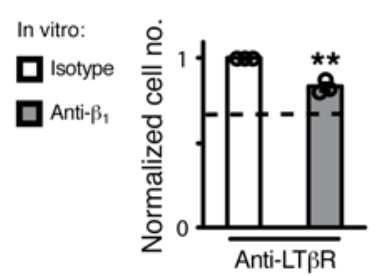

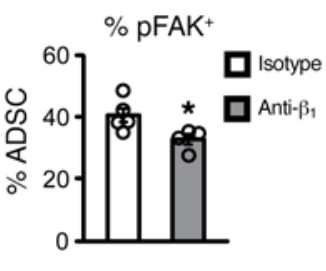

D Live human ADSC

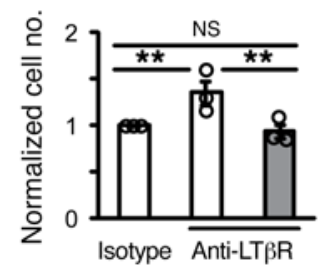

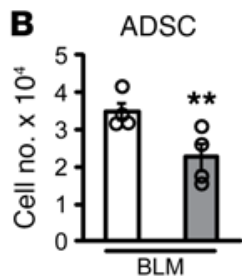

E $\% \mathrm{pFAK}^{+}$

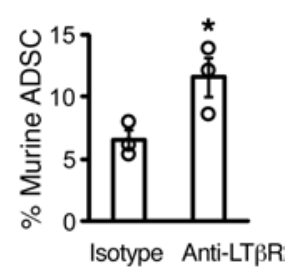

Figure 7. LT $\beta$ R signaling maintains ADSC survival via $\beta_{1}$ integrin activation. (A) Effect of $\beta_{1}$ integrin neutralization on ADSC pFAK. Mice treated with BLM over 22 days were treated with $50 \mu \mathrm{g}$ isotype control or neutralizing anti- $\beta$ integrin antibody 6 hours before analysis of skin. Left: Representative histogram of pFAK in ADSCs. Right: Percentage of ADSCs expressing pFAK. $n=4-5$ mice per condition over 2 experiments. (B) Effect of $\beta_{1}$ integrin neutralization on ADSC numbers. Mice treated with BLM over 21 to 32 days were treated with isotype control or neutralizing anti- $\beta_{1}$ integrin antibody 24 hours before analysis. Numbers are reported per 8-mm punch. $n=4$ mice per condition over 3 experiments. (C) Effect of $\beta_{1}$ integrin neutral-

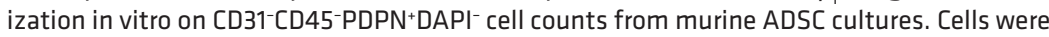
serum-starved in the presence of agonist anti-LT $\beta R$ and with isotype control or neutralizing anti- $\beta_{1}$ integrin antibody for 24 hours. Dashed line indicates baseline of cell survival in antiLT $\beta$ R experiments from Figure $5 \mathrm{H}$. (D) Effect of $\beta_{1}$ integrin neutralization on CD31-CD45-PD$\mathrm{PN}^{+} \mathrm{DAPI}^{-}$cell counts from human primary ADSC cultures. Cells were serum-starved in the absence or presence of isotype control or agonist anti-LT $\beta R$, with isotype control or neutralizing anti- $\beta_{1}$ integrin antibody for 48 hours. (E) Effect of LT $\beta R$ stimulation on percentage pFAK ${ }^{+}$ among CD31-CD45-PDPN $^{+}$cells from murine ADSC cultures. Cells were serum-starved in the presence of isotype control or agonist anti-LT $\beta R$ antibody for 24 hours. (C-E) Each symbol represents 1 of 3 experiments with 2 to 3 replicate wells per experiment. ${ }^{*} P<0.05$, ${ }^{*} P<0.01$ using 2-tailed unpaired Student's $t$ test. Error bars depict the SEM.

an accumulation of monocyte-derived cells (Supplemental Figure $7 \mathrm{~A}$ ) consistent with previous reports $(7,50)$. In contrast to the BLM model, however, DC numbers were unchanged in GVHD mice (Figure 6E), further supporting the idea that decreased DC numbers in the BLM model did not contribute to ADSC loss during fibrosis induction. The requirement for $\mathrm{BALB} / \mathrm{c}$ and $\mathrm{B} 10$. D2 genetic strains in the GVHD model precluded generation of $\mathrm{Ltb}^{-/}$mixed chimeras, so we injected LT $\beta \mathrm{R}-\mathrm{Ig}$ (48). LT $\beta \mathrm{R}-\mathrm{Ig}$ treatment resulted in a $50 \%$ loss of ADSCs (Figure 6F) without affecting $\mathrm{EpCAM}^{+}$or EpCAM-PDPN- populations (Supplemental Figure 7B). These findings were consistent with the idea of a DC-ADSC axis in multiple models of scleroderma skin fibrosis.

LT $\beta R$ signaling maintains $A D S C$ survival via $\beta_{1}$ integrin activation. We next asked how LT $\beta$ R signaling mediated ADSC survival. $\beta_{1}$ Integrin signaling through phosphorylation of focal adhesion kinase (FAK) can promote cell survival (51), and DCs maintain phosphorylated FAK (pFAK) in lymph node reticular cells (18). ADSCs in BLM-treated skin were $\mathrm{pFAK}^{+}$(Figure 7A), and treatment with neutralizing anti- $\beta_{1}$ integrin at a dose that mobilized neutrophils from bone marrow (52) (Supplemental Figure 8, A and B) reduced pFAK $^{\text {hi }}$ ADSCs by 6 hours (Figure 7A). By 24 hours, there was a $35 \%$ decrease in ADSC numbers (Figure 7B), similar to the effect of DC depletion or induced DC-specific LT $\beta$ deficiency (Figure 4F and Figure 5C). During serum starvation in vitro, anti- $\beta_{1}$ integrin reduced the LT $\beta R$-driven survival of murine and human ADSCs (Figure 7, C and D), and LT $\beta R$ stimulation increased pFAK (Figure 7E). These data suggested that LT $\beta R$ stimulation maintained ADSC survival via $\beta_{1}$ integrin activation.

$L T \beta R$ stimulation increases engraftment and therapeutic effect of injected ADSCs. Local injection of ADSCs or bulk adipose stromal-vascular cells that contain ADSCs has shown promising effects in scleroderma anecdotally and in uncontrolled clinical trials (53-55). Limited ADSC survival is felt to be an obstacle to optimal mesenchymal stromal cell therapies (15), and we found that the procedure itself of ADSC isolation caused about $30 \%$ of ADSCs to die (data not shown). Thus we asked whether engraftment and therapeutic efficacy of injected ADSCs could be augmented by addition of LT $\beta$ R stimulation to improve their survival. Control unfractionated skin cells or purified DWAT ADSCs from mCherry reporter mice were injected into fibrotic lesions of BLM-treated mice that were then treated without or with systemic agonist anti-LT $\beta R$ in a regimen that increased ADSC ICAM-1 (Figure 8A and Supplemental Figure 9A). ADSC plus anti-LT $\beta R$ treatment increased engraftment of mCherry ${ }^{+}$ ADSCs at 2 weeks after cell injection (Figure 8B). Host ADSCs showed a small increase in numbers and proliferation rate (Figure 8, C and D), suggesting progression toward a healthier state. DWAT thickness was doubled (Figure 8, E and F), reaching about half of the thickness found in homeostatic skin (compare Figure 8F with Figure 2B). More impressively, dermal thickness and collagen content were reduced by an amount approaching the magnitude by which they were increased with BLM treatment (compare Figure 8, F and G, with Figure 2, B and C). Notably, whole skin plus anti-LT $\beta R$ and ADSC plus isotype conditions did not affect ADSCs, DWAT, or dermal fibrosis (Figure $8, C-G$ ), suggesting that LT $\beta R$ stimulation or ADSCs alone were not sufficient to induce skin improvement. Remarkably, 4 of the 12 BLM-induced gene expression changes were reversed with combined ADSC plus anti-LT $\beta R$ treatment, and Wnt-related genes also trended toward reversal (Figure 8H, underlined genes, and Supplemental Table 1). Notably, genes that were not affected by BLM treatment were also not altered by combined ADSC plus anti-LT $\beta$ R treatment (Figure 8H), suggesting that ADSC plus anti-LT $\beta$ R treatment had beneficial effects by reversing aspects of the BLM-induced changes rather than by superimposing additional changes. Despite partial DWAT recovery, perilipin, which correlated well with DWAT loss with BLM and then further with DC depletion (Figure 4, C and H), was not consistently upregulated (Figure $8 \mathrm{H}$ ), potentially reflecting the small magnitude of DWAT change and the variability among the small number of individual animals (Figure 8F). Although some mCher$\mathrm{ry}^{+}$cells could be identified that expressed leptin (Supplemental Figure 9B), suggesting differentiation into adipocytes, gene 

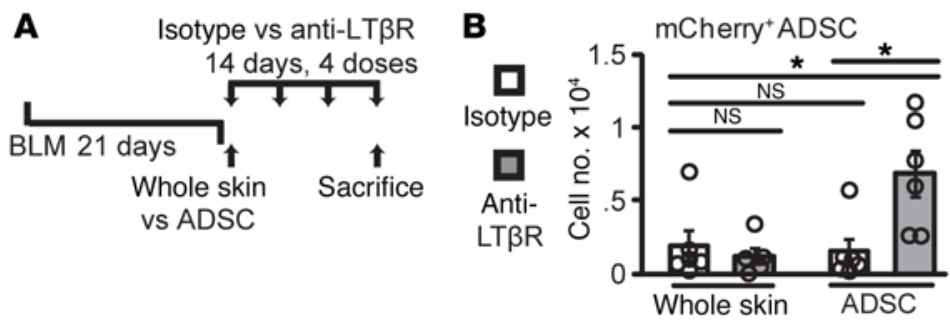
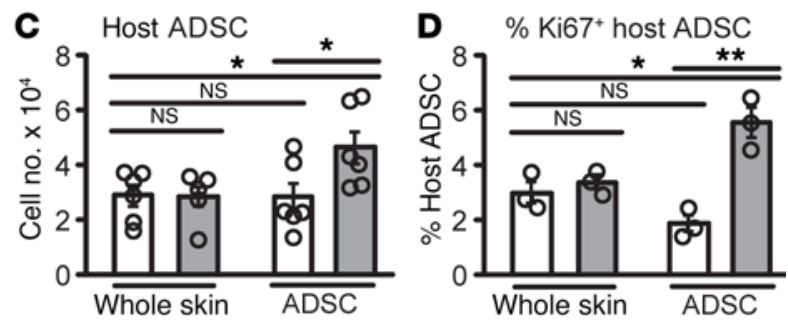

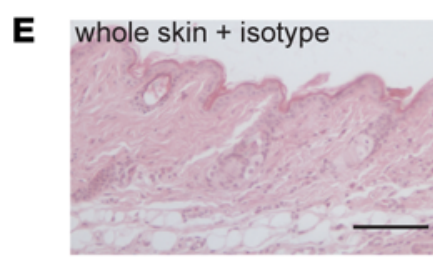

$\mathbf{F}$

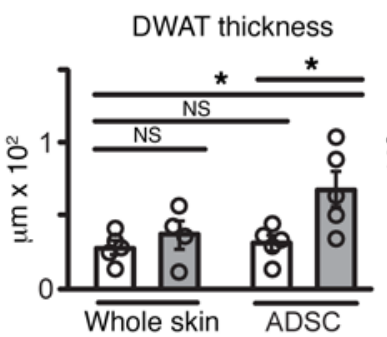

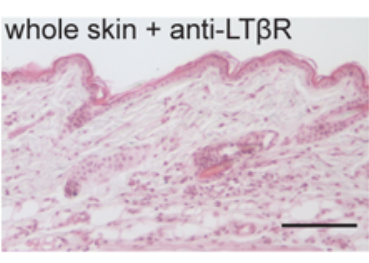

Dermal thickness

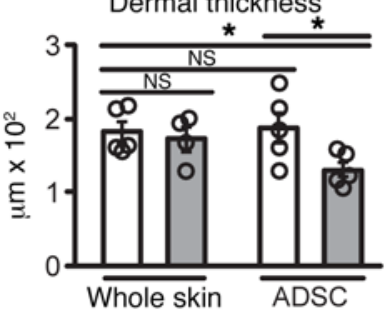

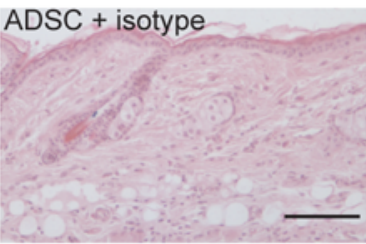
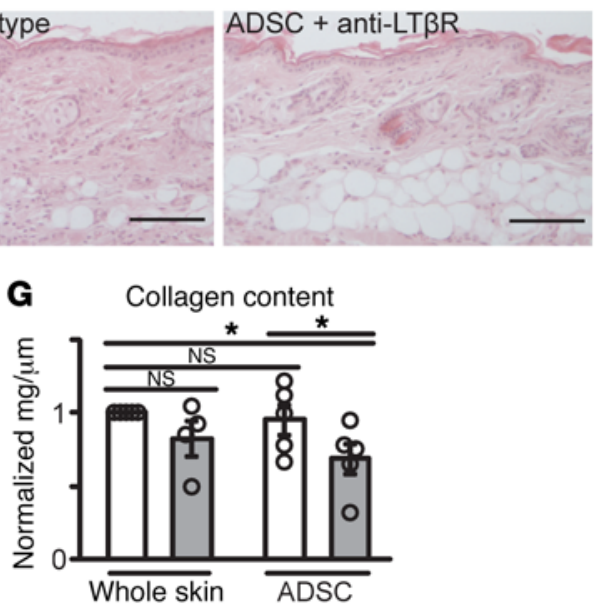
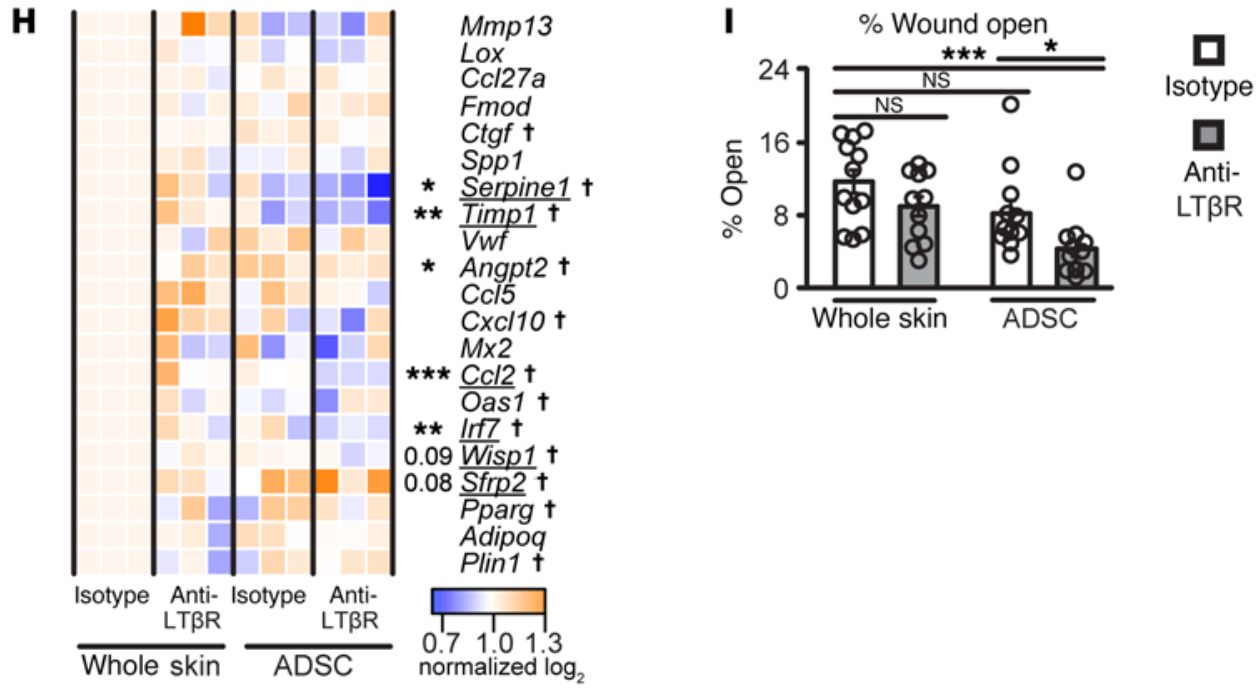

Figure 8. LT $\boldsymbol{\beta R}$ stimulation increases engraftment and therapeutic effect of injected ADSCs. (A) Experimental schematic for B-H. Mice were treated with BLM over 21 days, and either whole skin cells or ADSCs from mCherry reporter mice were injected into fibrotic lesions 2 days after BLM cessation. Isotype control or agonist anti-LT $\beta R$ was given i.p. at the time of cell injection and then twice a week for 14 days before skin analysis ( 2 doses of $20 \mu g$ and then 2 doses of 10 $\mu$ g). (B) mCherry ${ }^{+}$ADSC numbers per 8-mm punch. (C) Host ADSC numbers per punch. (B and C) $n=5-6$ mice per condition over 6 experiments. (D) Percentage of host ADSCs that are Ki67 ${ }^{+} . n=3$ mice per condition over 3 experiments. (E) Representative H\&E-stained sections. Scale bars: 100 $\mu$ m. (F) DWAT and dermal thicknesses. (G) Collagen content normalized to whole skin plus isotype condition. (E-G) $n=4-5$ mice per condition over 5 experiments. (H) Relative mRNA expression of indicated genes using NanoString. $n=3$ mice per condition over 3 experiments. Asterisks denote statistical significance of differences between the whole skin plus isotype and the ADSC plus anti-LT $\beta \mathrm{R}$ conditions. ${ }^{\dagger}$ Cenes that changed with BLM treatment in Figure $4 \mathrm{H}$. Underlined genes change in the direction opposite that seen with BLM treatment. See Supplemental Table 1 for additional analysis. (I) Mice were treated as in A-H before full-thickness wounding, with no further treatment over 14 days before skin analysis. $n=11-12$ wounds in 6 mice per condition over 6 experiments. ${ }^{*} P<0.05$, ${ }^{*} P<0.01$, ${ }^{* *} P<0.001$ using 2-tailed unpaired Student's $t$ test. Error bars depict the SEM.

expression results suggested that combined ADSC plus anti-LT $\beta R$ treatment may have had beneficial effects at least in part independent of DWAT recovery. Consistent with the histologic and gene expression data, wound healing was improved with combined
ADSC plus anti-LT $\beta R$ treatment (Supplemental Figure 9C and Figure 8I). Together these results suggested that providing LT $\beta R$ stimulation to improve survival of injected ADSCs led to reduced dermal fibrosis and improved skin state and function. 


\section{Discussion}

Our results suggest a scenario whereby ADSC survival is differentially regulated in fibrosis, and a DC-ADSC axis emerges in fibrotic skin: At homeostasis, ADSCs depend on factors other than DCs. During fibrosis induction, many ADSCs die, potentially because of loss of homeostatic survival factors as their DWAT niche atrophies. In established fibrosis, the remaining ADSCs have a partial dependence on DCs for their survival. This scenario points to DC-mediated ADSC survival as a stress survival mechanism. Consistent with the idea that ADSCs are under stress in fibrosis, ADSCs showed changes in state over the course of fibrosis development, with transient upregulation of proliferation and upregulation of PDPN. This parallels findings in lymph node reticular cells, which rely on a DCLT $\beta \mathrm{R}-\beta_{1}$ integrin axis for survival in inflamed but not homeostatic nodes (18). Furthermore, dependence on $\beta_{1}$ integrins, which are used by tumor cells for chemoresistance (50), further suggests that ADSCs are in a stressed state in fibrotic skin. That LT $\beta$ R stimulation was not sufficient to prevent early ADSC death during fibrosis induction suggests that this DC-dependent phenotype arises late in fibrosis development. Additional attributes of this state, the exact timing of the emergence of DC dependence, and the extrinsic modulators involved, whether lack of homeostatic survival factors, the stiffening of the matrix, or others, will require further exploration.

We showed that DCs maintained ADSC survival via LT $\beta$ and LT $\beta$ R-mediated $\beta_{1}$ integrin activation in ADSCs, suggesting that in fibrotic skin, LT $\beta$ R on ADSCs maintains $\beta_{1}$ integrin activation and consequent cell-matrix adhesion at levels needed for cell survival. The use of this pathway by both lymph node reticular cells and ADSCs may in part reflect a shared lineage, as adipocyte progenitors or adipose PDGFR $\beta^{+}$mesenchymal cells can differentiate into lymph node reticular cells $(20,56)$. However, a dependence on integrins when stressed by cytokines or chemotherapy occurs in multiple cell types $(51,57)$, and many mesenchymal and epithelial cells express LT $\beta R$ (47), suggesting the possibility of more widespread employment of the LT $\beta R / \beta_{1}$ integrin pathway, whether or not DCs are the source of LT $\beta$ R ligands.

In addition to loss of ADSCs, DC depletion over 14 days in fibrotic skin led to further DWAT loss along with gene expression and wound healing changes indicative of further skin injury. The loss of ADSCs likely contributed to the further reduction in DWAT and perilipin gene expression by eliminating adipocyte-forming cells. Whether ADSC loss also contributed to changes in gene expression and impaired wound healing is less clear. DC depletion-induced ADSC loss preceded gene expression changes, as these changes were not evident after 2 days of DC depletion and associated ADSC loss (Chia and Lu, unpublished observations). Direct targeting of ADSCs in the future will be needed to better understand the distinct consequences of DC versus ADSC loss to the skin. Interestingly, FLT3 ligand-dependent DCs have been implicated in resolution of fibrosis in the reversible carbon tetrachloride-induced hepatic fibrosis model (58), suggesting the possibility that DCs could play a similar role in maintaining regenerative cells in other organs. Additionally, IRF4-dependent DCs were recently shown to maintain normal permeability of collecting lymphatics in subcutaneous adipose tissue (59). Collectively along with our studies of DCs in lymph node reticular cell survival during ongoing immune responses (18), these findings suggest that DCs are guardians of tissue function.
Although the LT $\beta$ staining in DCs was modest, the mixed chimera experiments established that LT $\beta$ mediated the DC effect on ADSC survival, as only ZBTB46 ${ }^{+}$DCs and not other cell types become LT $\beta$-deficient upon DT injection. DCs maintained the survival of about $35 \%-40 \%$ of ADSCs in fibrotic skin, leaving room for additional mechanisms of regulation. Although our results indicated that other mononuclear phagocyte populations did not mediate the DC effects on ADSC survival, they do not preclude a role for these other populations in contributing to ADSC survival independent of DCs.

Remarkably, although BLM-induced skin fibrosis is considered irreversible (23), fibrosis and other aspects of BLM-induced injury were partially reversed by simply administering exogenous ADSCs with a survival signal. Our results suggest that the loss of DWAT ADSCs in fibrotic skin may contribute to the development or maintenance of fibrosis, and that ADSC therapy may serve at least in part to replenish the deficiency of reparative ADSCs in fibrotic skin. It will be important to further understand the role of ADSCs in fibrosis protection and examine whether ADSC replenishment should be considered a distinct therapeutic goal, perhaps in concert with other therapeutic approaches such as targeting of profibrotic pathways $(60,61)$.

Our results suggest provision of LT $\beta R$ stimulation as a strategy to improve mesenchymal stromal cell therapy. This strategy in our experiments had partial effects, and further investigations are needed to understand the extent to which this strategy can be further optimized. Clinically, a partial effect with one strategy may be sufficient when combined with other therapeutic strategies. In addition to treating skin fibrosis, LT $\beta$ R stimulation could potentially be used for other aspects of scleroderma. ADSCs and bone marrow mesenchymal stromal cells are being investigated for idiopathic pulmonary fibrosis (ClinicalTrials.gov, NCT02135380, NCT01919827), and have been proposed for scleroderma pulmonary fibrosis, a significant cause of mortality in systemic sclerosis $(62,63)$. Additionally, mesenchymal stromal cell therapy is being investigated for other systemic diseases such as GVHD, rheumatoid arthritis, and lupus, as well as conditions requiring local bone and muscle repair (15, 64). Thus, provision of LT $\beta$ R stimulation could potentially be used to improve therapeutic effectiveness of mesenchymal stromal cells generally and in a wide range of diseases.

\section{Methods}

Supplemental Methods are available online with this article; doi:10.1172/JCI85740DS1.

\section{Mice}

Female mice 6-14 weeks old were used unless otherwise indicated. C57BL/6 mice were from The Jackson Laboratory (JAX), Taconic Farms, the National Cancer Institute (NCI), or our own breeding colony. Congenic CD $45.1^{+}$mice were from NCI or our own breeding colony. $\mathrm{zDC}^{\mathrm{GFP} / \mathrm{GFP}}$ mice (28) were on a $129 \mathrm{~S}$ background, and $129 \mathrm{~S}$ recipients for $\mathrm{zDC}^{\mathrm{GFP} / \mathrm{GFP}} \rightarrow \mathrm{WT}$ chimeras were all from JAX. $\mathrm{zDC}^{\mathrm{DTR} /+}, \mathrm{zDC}^{\mathrm{DTR} /+}$ CD 45.1 $1^{+/-} \mathrm{CD} 45.2^{+/-}, \mathrm{Ltb}^{-/}$, and $\mathrm{Ltb}^{-/-} \mathrm{Ragl}^{-/-}$mice have been previously described $(18,29,65)$. BALB/c and BALB/c Rag2 $2^{--}$mice were from Taconic Farms. B10.D2- $H c^{1} H 2^{d} H 2-T 18^{c} / \mathrm{nSnJ}$ (B10.D2) were from JAX, and $\mathrm{B} 6(\mathrm{Cg})-\mathrm{Ty} r^{-2 J} \mathrm{Tg}$ (UBC-mCherry) $1 \mathrm{Phbs} / \mathrm{J}$ (mCherry) mice were from JAX and bred at our facility. 


\section{Mouse treatments}

$B L M$ model. The lower backs of mice not in anagen were shaved, and $100 \mu \mathrm{l}$ of BLM $(0.2 \mathrm{mg} / \mathrm{ml}$ in PBS; Enzo Life Sciences) was injected s.c. into each of 3 adjacent sites $5 \mathrm{~d} /$ wk over the duration indicated. PBS was administered as a control.

Rat anti-LT $\beta$ R agonist antibody (clone eBio3C8, Affymetrix/eBioscience) (47) or rat IgG1 isotype control (Affymetrix/eBioscience) was given by retro-orbital injection at indicated doses and timing.

Anti- $\beta$ integrin antibody (18) (anti-CD29, clone HM $11-1$, BioLegend) or Armenian hamster isotype control (BioLegend) was given by tail vein injection.

Systemic sclerosis-GVHD model. Twenty million splenocytes from $\mathrm{BALB} / \mathrm{c}$ (congenic) or B10.D2 mice were injected retro-orbitally into BALB/c Rag2 ${ }^{-/}$recipients, and mice were sacrificed after 22-23 days. Indicated mice received i.p. injections of LT $\beta$ R-Ig or control mouse IgG1 clone MOPC21 (Biogen).

\section{Bone marrow chimeras and DT administration}

Bone marrow chimeras were generated as previously described (18). For DC depletion, the first DT dose was 250-300 ng via i.p. injection, and subsequent doses were 125-150 ng.

\section{Flow cytometric staining and sorting}

To generate single-cell suspensions of skin for flow cytometry, we added dispase to our lymph node digestion protocol (18). Back skin was removed, and 8-mm biopsy punches were obtained from the BLMaffected region. Skin was finely minced before digestion in type II collagenase (616 U/ml; Worthington Biochemical Corp.) and dispase (2.42 U/ml; Life Technologies), and then processed as described (18). Cells were counted using a Z1 Coulter Counter (Beckman Coulter).

For skin fractionation experiments, we used angled forceps to separate the DWAT from the epidermis/dermis of 8-mm punches. For inguinal fat pads, we discarded the inguinal lymph node and proceeded as for skin.

Antibodies and staining reagents included CCR2-APC (Allophycocyanin) (R\&D Systems), mCherry-Alexa 488 (Life Technologies), and SMA-FITC (Sigma-Aldrich). From BD Biosciences were B220-biotin, CD45-FITC, and Ki67-Alexa 647. From Affymetrix/eBioscience were pan-NK-bio (also called CD49b), CD31-FITC, and CD34-biotin. From BioLegend were CD3-biotin, Ly6G-biotin, Ly6G-Alexa 700, CD45Alexa 700, CD45-PCPCy5.5, CD45.1-Alexa 700, CD45.2-APC-Cy7, CD11c-APC-Cy7, CD24-PCPCy5.5, CD11b-BV570, IAb-PE (Phycoerythrin), IAb-FITC, Ly6C-PECy7, Ly6C-FITC, CD64-APC, CD64-PE, CD31-PECy7, EpCAM-PECy7, EpCAM-APC, EpCAM-biotin, PDPNPE, PDPN-APC, Thy1.2-APC, Thy1.2-Alexa 700, Sca1-APCCy7, ICAM-FITC, and CD29. Unconjugated CD29 was detected with anti-Armenian hamster-biotin (Jackson ImmunoResearch). Biotinylated antibodies were detected with streptavidin conjugated to Pacific Blue or APC, and dead cells were identified with DAPI (all from Life Technologies).

TUNEL staining (Roche) was performed as previously described (18).

For pFAK (18), cells were processed and stained in the presence of $2 \mathrm{mM}$ sodium orthovanadate (Sigma-Aldrich) from the final 15 minutes of enzymatic digestion onward. After digestion, cells were immediately fixed in 1\% paraformaldehyde for 20 minutes on ice followed by extracellular staining with eBioscience fixation/permeabilization reagent for 30 minutes at room temperature. The cells were incubated with Fc block for 10 minutes and then with antibody against pFAK at Y397 (ABT135, Millipore) or rabbit isotype control (BD Biosciences) for 40 minutes at room temperature. Primary antibodies were detected with anti-rabbit Alexa Fluor 647 (Jackson ImmunoResearch).

Intracellular LT $\beta$ staining by flow cytometry was performed with rabbit anti-LT $\beta$ antibody (Santa Cruz Biotechnology) for 40 minutes at room temperature after BD Biosciences fixation/permeabilization for 30 minutes at room temperature, and detected with anti-rabbit Alexa 647.

Cells were analyzed using FACSCanto or FACSCalibur (both BD Biosciences) and FlowJo software (Tree Star).

For flow cytometric sorting of CD11b ${ }^{+}$DCs, DWAT from the trunk and inguinal fat pads (inguinal lymph node discarded) of 2 mice per experiment was digested as above, except for increased type II collagenase $(924 \mathrm{U} / \mathrm{ml})$, dispase $(3.63 \mathrm{U} / \mathrm{ml})$, and DNase1 $(0.24 \mathrm{mg} / \mathrm{ml})$ to accommodate the tissue volume, and stained. Cells were sorted using a BD FACSVantage. Purity of sorted DCs was at least 95\%.

\section{Cell calculations from flow cytometry}

To calculate cell number, the percentage of the total gated population was multiplied by the total cell count from the Coulter Counter. For normalized values, the control sample was set to 1 , and the value from the experimental sample was normalized. For experiments with more than 1 control sample, the control values were averaged, and the individual control and experimental samples were calculated relative to this average value.

\section{Histology, immunofluorescence staining and analyses}

For murine histology, skin was fixed in Z-Fix (Anatech), then dehydrated and embedded in paraffin. Seven-micrometer sections were cut, rehydrated, and stained with H\&E (Electron Microscopy Sciences). DWAT and dermal thicknesses were measured by a blinded observer using ImageJ software (NIH). Adipocytes were enumerated based on their morphology on H\&E-stained sections.

For visualization of $\mathrm{GFP}^{+}$cells in $\mathrm{zDC}^{\mathrm{GFP} / \mathrm{GFP}}$ chimera skin, paraffin-embedded $7-\mu \mathrm{m}$ sections were rehydrated before antigen retrieval at $60^{\circ} \mathrm{C}$ in $10 \mathrm{mM}$ citrate buffer, $\mathrm{pH} 6.0$, for 20 hours and then stained as indicated. GFP was detected with rabbit anti-GFP (Abcam) and anti-rabbit rhodamine (Jackson ImmunoResearch). For mCher$\mathrm{ry}^{+}$cell localization, skin was fixed in $4 \%$ paraformaldehyde on ice for 60 minutes, sucrose-impregnated overnight, and frozen in OCT compound. Ten-micrometer sections were fixed in cold acetone for 10 minutes, and stained as indicated. Additional antibodies used for murine immunofluorescence staining were anti-mCherry-Alexa 594 (Life Technologies) and anti-leptin (R\&D Systems) detected with antigoat-Alexa 647 (Jackson ImmunoResearch). Nuclei were visualized with DAPI (Life Technologies). GFP ${ }^{+}$cells were counted using ImageJ software and normalized to the DWAT or dermal area.

Human formalin-fixed paraffin-embedded sections from lesional forearm skin of 3 patients with early diffuse systemic sclerosis were rehydrated before antigen retrieval at $60^{\circ} \mathrm{C}$ in $10 \mathrm{mM}$ citrate buffer, $\mathrm{pH}$ 6.0, for 4 hours and stained with mouse anti-human CD1c (clone $2 \mathrm{~F} 4$, Abcam) or mouse IgG1 isotype control (R\&D Systems), followed by anti-mouse IgG1-biotin (Jackson ImmunoResearch), and detected with streptavidin-rhodamine (Jackson ImmunoResearch). Anti-perilipin (Abcam) was detected with anti-rabbit-Alexa 647 (Jackson ImmunoResearch). $\mathrm{CD} 1 \mathrm{c}^{+}$cells were counted using Image J software and normalized to the DWAT (perilipin-containing) or dermal area. 
Immunofluorescence images were captured using a Nikon Eclipse NI-E Fluorescence Upright microscope coupled to a Zyla sCMOS camera (Andor Technology) and analyzed using NIS Elements software (Nikon) and ImageJ (NIH).

\section{Collagen quantification}

Skin collagen content was quantified using QuickZyme Biosciences Total Collagen Kit (the Netherlands) according to the manufacturer's protocol. Five 7- $\mu \mathrm{m}$ sections from paraffin-embedded samples were used for collagen extraction, and the next serial section was placed on a slide and H\&E-stained. Collagen was normalized to the length of this H\&E-stained serial section. Section lengths were measured by a blinded observer using ImageJ software.

\section{mRNA quantification}

Skin was stored in RNAlater (Qiagen Sciences) at $-80^{\circ} \mathrm{C}$ from time of harvest until RNA extraction, which was performed using Qiagen RNeasy Mini Kit. NanoString was performed according to the manufacturer's protocol using $75 \mu \mathrm{g}$ total RNA (NanoString Technologies). NanoString gene expression was normalized to Actb, Api5, Eef1a1, Rpl36al, and Sf3b2. Tgfb1, Sox9, and Adipoq mRNA was measured using quantitative real-time PCR. iScript cDNA synthesis kit (Bio-Rad) was used to generate cDNA. iQ SYBR Green Supermix kit (Bio-Rad) was used for real-time PCR on a BioRad MyiQ thermal cycler. Quantitative PCR (qPCR) gene expression was quantified relative to Gapdh.

Primer sequences. Primer sequences were as follows: Adipoq: forward,AAGAAGGACAAGGCCGTTCTCTT; reverse, GCTATGGGTAGTTGCAGTCAGTT; Gapdh: forward, ATGTGTCCGTCGTGGATCTGA; reverse, TTGAAGTCGCAGGAGACAACCT; $T g f b 1$ : forward, GCAACAATTCCTGGCGTTACC; reverse, CCСTGTATTCCGTCTCCTTGGT; Sox9: forward, AAGCTCTGGAGGCTGCTGAACGAG; reverse, CGGCCTCCGCTTGTCCGTTCT.

\section{Wound healing}

Full-thickness wounds were created by gathering of lesional fibrotic skin and excision of a $4-\mathrm{mm}$ punch on either side of midline. Area was measured by tracing onto a glass slide at the time of wounding, and again after sacrifice, but before dissection. Slides were scanned and area calculated with ImageJ.

\section{Primary murine cell culture}

For ADSC cultures, inguinal fat pads were digested as for flow cytometric analysis, resuspended in DMEM/F-12 50/50 (Thomas Scientific) containing $2 \mathrm{mM}$ L-glutamine, $50 \mathrm{IU} / \mathrm{ml}$ penicillin/streptomycin, $15 \mathrm{mM}$ HEPES, and $15 \%$ FCS, and plated at 1.5 million to 2 million cells per well in 24-well tissue culture plates. After 24 hours, cultures were washed of nonadherent cells, and medium changed every 2 days until confluence at about day 6. Cultures were at least 97\% CD31-CD45 and about $94 \% \mathrm{PDPN}^{+} \mathrm{Sca}^{+}$. For assays, cells were plated at 15,000 cells per well in 96-well tissue culture plates. After 24 hours, cells were washed twice and changed to medium without FCS, with $1 \mu \mathrm{g} / \mathrm{ml}$ goat anti-LTßR (18) or goat IgG (R\&D Systems), with or without $1 \mu \mathrm{g} / \mathrm{ml}$ anti- $\beta_{1}$ integrin antibody (18) or hamster IgG as indicated.

For cocultures with sorted CD11b DCs, inguinal fat pad ADSC cultures were plated at 15,000 cells per well. After 4-6 hours, 10,000-15,000 $\mathrm{CD} 11 \mathrm{~b}^{+}$DCs were added. After 24 hours, medium was changed to serumfree medium without or with $10 \mu \mathrm{g} / \mathrm{ml} \operatorname{LT} \beta \mathrm{R}-\mathrm{Ig}(18,48)$ as indicated.
For adipocyte differentiation, DWAT was digested as for flow cytometric analysis or in $200 \mu \mathrm{g}$ Liberase TL (Roche) and DNase1 $(0.24 \mathrm{mg} /$ $\mathrm{ml}$ ), and resuspended in DMEM containing 0.5\% BSA and 2 mM EDTA. ADSCs were isolated by CD31, CD45, and EpCAM depletion, followed by $\mathrm{Sca}^{+}$selection by magnetic selection (Miltenyi Biotec) to about $94 \%$ purity. In some experiments, DWAT ADSCs were isolated from whole skin in parallel with EpCAM-PDPN- cells via FACS sorting, both to about $96 \%$ purity. Cells were plated at 80,000-100,000 cells per well in 96-well tissue culture plates, and DMEM/F-12 with $10 \%$ FCS was changed daily for 3 days before addition of 3-isobutyl-1-methylxanthine (IBMX, $100 \mu \mathrm{g} / \mathrm{ml}$; Sigma-Aldrich), dexamethasone (0.25 $\mu \mathrm{M}$; Sigma-Aldrich), insulin (1 $\mu \mathrm{g}$ / $\mathrm{ml}$; Sigma-Aldrich), and rosiglitazone ( $1 \mu \mathrm{M})$ (66). After 3 days, medium was changed to include only insulin and refreshed every other day for 6 days before Oil Red O staining (Sigma-Aldrich). Oil Red O-stained cultures were counterstained with hematoxylin, and the number of Oil Red $\mathrm{O}^{+}$cells was divided by the total number of hematoxylin-positive nuclei.

ADSCs were differentiated into osteoblasts using the StemPro Osteogenesis Differentiation Kit (Thermo Fisher Scientific) for 21 days before fixation and staining with Alizarin Red, $\mathrm{pH} 4.2$ (Sigma-Aldrich). The stained cells were photographed. The alizarin was then solubilized in $10 \%$ acetic acid for 30 minutes followed by neutralization with $10 \%$ ammonium hydroxide, and colorimetric readings were taken at $450 \mathrm{~nm}$.

ADSCs were differentiated into chondrocytes using StemPro Chondrogenesis Differentiation Kit (Thermo Fisher Scientific) for 22 days. RNA was extracted using an RNeasy Micro Kit (Qiagen), and Sry-related high-mobility-group box transcription factor 9 (Sox9) and aggrecan (Acan) (67) were measured by quantitative real-time PCR. Parallel wells were fixed and stained with Alcian blue, pH 2.5 (Millipore), before being photographed.

\section{Primary human cell culture}

Human primary ADSCs were purchased from Lonza. Cells were expanded according to the manufacturer's specifications and used between passages 1 and 2. Cells were plated at 10,000 cells per well in 96-well plates in ADSC Basal medium with growth supplement (Lonza). After 24 hours, cells were washed twice with MEM- $\alpha$ (Life Technologies) containing $2 \mathrm{mM}$ L-glutamine, $50 \mathrm{IU} / \mathrm{ml}$ penicillin/streptomycin, and $10 \mathrm{mM}$ HEPES without FCS, then treated with $9 \mu \mathrm{g} / \mathrm{ml}$ mouse antihuman LT $\beta R$ (clone 31G4D8, BioLegend) (49) or mouse IgG2b isotype control (BioLegend), with or without $1 \mu \mathrm{g} / \mathrm{ml}$ mouse anti-human $\beta_{1}$ integrin antibody (clone P5D2, BioLegend) or mouse IgG1 isotype (BioLegend) as indicated for 48 hours. Cells were then trypsinized and stained for FACS using antibodies for anti-human CD31-PECy7, CD45-FITC, Thy1-PCPCy5.5, and PDPN-PE (all BioLegend).

\section{ADSC isolation and injection}

Female or male mCherry donors were used as cell donors for injection. Whole skin cells were digested as for cell sorting, and DWAT ADSCs were isolated as for in vitro differentiation to about $94 \%$ purity and 3.9- to 5.4-fold enrichment of purified ADSCs over whole skin, where ADSCs typically constitute $17 \%$ of cells from whole skin $( \pm 4 \%, n=9)$. Cells were counted with trypan blue on a hemacytometer, and 25,000 cells were injected intradermally per BLM injection site.

\section{Statistics}

Statistical significance was determined using 2-tailed unpaired Student's $t$ test. A $P$ value less than 0.05 was considered significant. 


\section{Study approval}

All animal procedures were performed in accordance with the regulations of the Institutional Animal Care and Use Committee at the Hospital for Special Surgery.

Punch biopsies from scleroderma patients were taken in accordance with a protocol approved by the Institutional Review Board at the Hospital for Special Surgery. All subjects gave written informed consent prior to inclusion.

\section{Author contributions}

JJC, TZ, SC, DD, ST, RL, JLB, and CC designed, performed, and interpreted experiments. CMM interpreted experiments. NHR, TEM, JLB, and SR provided reagents. RFS and JKG provided patient skin sample slides. NHR, TEM, RL, JLB, and JKG provided critical input during manuscript development and writing. TTL designed, supervised, and interpreted experiments. JJC and TTL wrote the paper.

\section{Acknowledgments}

We thank Mihaela Stevanovic and Sergei Rudchenko of the Hospital for Special Surgery/Cornell flow cytometry core facility for assistance with cell sorting, Michael Olferiev, Orla O'Shea, and
Inez Rogatsky's laboratory for helpful discussions and reagents, and Will Shipman and Dennis Chia for comments on the manuscript. This work was supported by MSTP T32GM007739 from National Institute of General Medical Sciences/NIH to the Weill Cornell/Rockefeller/Sloan Kettering Tri-Institutional MD-PhD Program (to JJC) and T32AI007621 to the Immunology and Microbial Pathogenesis Program of Weill Cornell Graduate School of Medical Sciences (to JJC); National Institute of Allergy and Infectious Diseases/NIH R01AI079178, National Center for Advancing Translational Sciences/NIH 5UL1RR024996, the Alliance for Lupus Research, the St. Giles Foundation, and an O'Neill Foundation grant from the Barbara Volcker Center for Women and Rheumatic Diseases (all to TL); and a grant from Connecticut Innovations Inc., 13-SCA-Yale-27 (to NHR). The content of this work is solely the responsibility of the authors and does not necessarily represent the official views of the National Institutes of Health.

Address correspondence to: Theresa T. Lu, Hospital for Special Surgery, 535 East 70th Street, New York, New York 10021, USA. Phone: 212.774.2532; E-mail: lut@hss.edu.
1. Krieg T, Takehara K. Skin disease: a cardinal feature of systemic sclerosis. Rheumatology (Oxford). 2009;48 Suppl 3:iii14-iii18.

2. Fett N, Werth VP. Update on morphea: part I. Epidemiology, clinical presentation, and pathogenesis. J Am Acad Dermatol. 2011;64(2):217-228.

3. Driskell RR, Jahoda CA, Chuong CM, Watt FM, Horsley V. Defining dermal adipose tissue. Exp Dermatol. 2014;23(9):629-631.

4. Fleischmajer R, Damiano V, Nedwich A. Scleroderma and the subcutaneous tissue. Science. 1971;171(3975):1019-1021.

5. Wu M, Melichian DS, Chang E, Warner-Blankenship M, Ghosh AK, Varga J. Rosiglitazone abrogates bleomycin-induced scleroderma and blocks profibrotic responses through peroxisome proliferator-activated receptor-gamma. Am J Pathol. 2009;174(2):519-533.

6. Marangoni RG, et al. Myofibroblasts in murine cutaneous fibrosis originate from adiponectin-positive intradermal progenitors. Arthritis Rheumatol. 2015;67(4):1062-1073.

7. Ruzek MC, Jha S, Ledbetter S, Richards SM, Garman RD. A modified model of graft-versushost-induced systemic sclerosis (scleroderma) exhibits all major aspects of the human disease. Arthritis Rheum. 2004;50(4):1319-1331.

8. Wei J, et al. Canonical Wnt signaling induces skin fibrosis and subcutaneous lipoatrophy: a novel mouse model for scleroderma?. Arthritis Rheum. 2011;63(6):1707-1717.

9. Stawski L, Han R, Bujor AM, Trojanowska M. Angiotensin II induces skin fibrosis: a novel mouse model of dermal fibrosis. Arthritis Res Ther. 2012;14(4):R194

10. Gerber EE, et al. Integrin-modulating therapy prevents fibrosis and autoimmunity in mouse models of scleroderma. Nature. 2013;503(7474):126-130.

11. Martins V, Gonzalez De Los Santos F, Wu Z, Capelozzi V, Phan SH, Liu T. FIZZ1-induced myofibroblast transdifferentiation from adipocytes and its potential role in dermal fibrosis and lipoatrophy. Am J Pathol. 2015;185(10):2768-2776.

12. Cawthorn WP, Scheller EL, MacDougald OA. Adipose tissue stem cells meet preadipocyte commitment: going back to the future. J Lipid Res. 2012;53(2):227-246.

13. Ogawa R, Mizuno H, Watanabe A, Migita M, Hyakusoku H, Shimada T. Adipogenic differentiation by adipose-derived stem cells harvested from GFP transgenic mice-including relationship of sex differences. Biochem Biophys Res Commun. 2004;319(2):511-517.

14. Ogawa R, Mizuno H, Watanabe A, Migita M, Shimada T, Hyakusoku H. Osteogenic and chondrogenic differentiation by adipose-derived stem cells harvested from GFP transgenic mice. Biochem Biophys Res Commun. 2004;313(4):871-877.

15. Grayson WL, Bunnell BA, Martin E, Frazier T, Hung BP, Gimble JM. Stromal cells and stem cells in clinical bone regeneration. Nat Rev Endocrinol. 2015;11(3):140-150.

16. Driskell RR, et al. Distinct fibroblast lineages determine dermal architecture in skin development and repair. Nature. 2013;504(7479):277-281.

17. Merad M, Sathe P, Helft J, Miller J, Mortha A. The dendritic cell lineage: ontogeny and function of dendritic cells and their subsets in the steady state and the inflamed setting. Annu Rev Immunol. 2013;31:563-604.

18. Kumar V, et al. A dendritic-cell-stromal axis maintains immune responses in lymph nodes. Immunity. 2015;42(4):719-730.

19. Litvinov SV, Velders MP, Bakker HA, Fleuren GJ, Warnaar SO. Ep-CAM: a human epithelial antigen is a homophilic cell-cell adhesion molecule. JCell Biol. 1994;125(2):437-446.

20. Bénézech $C$, et al. Lymphotoxin- $\beta$ receptor signaling through NF- $\mathrm{kB} 2-$ RelB pathway reprograms adipocyte precursors as lymph node stromal cells. Immunity. 2012;37(4):721-734.
21. Parekkadan B, et al. Aire controls mesenchymal stem cell-mediated suppression in chronic colitis. Mol Ther. 2012;20(1):178-186.

22. Festa E, et al. Adipocyte lineage cells contribute to the skin stem cell niche to drive hair cycling. Cell. 2011;146(5):761-771.

23. Batteux F, Kavian N, Servettaz A. New insights on chemically induced animal models of systemic sclerosis. Curr Opin Rheumatol. 2011;23(6):511-518.

24. Sargent JL, et al. Identification of optimal mouse models of systemic sclerosis by interspecies comparative genomics. Arthritis Rheumatol. 2016;68(8):2003-2015.

25. Akamatsu T, et al. Direct isolation of myofibroblasts and fibroblasts from bleomycin-injured lungs reveals their functional similarities and differences. Fibrogenesis Tissue Repair. 2013;6(1):15.

26. Dulauroy S, Di Carlo SE, Langa F, Eberl G, Peduto L. Lineage tracing and genetic ablation of ADAM12(+) perivascular cells identify a major source of profibrotic cells during acute tissue injury. Nat Med. 2012;18(8):1262-1270.

27. Tamoutounour $\mathrm{S}$, et al. Origins and functional specialization of macrophages and of conventional and monocyte-derived dendritic cells in mouse skin. Immunity. 2013;39(5):925-938.

28. Satpathy AT, et al. Zbtb46 expression distinguishes classical dendritic cells and their committed progenitors from other immune lineages. J Exp Med. 2012;209(6):1135-1152.

29. Meredith MM, et al. Expression of the zinc finger transcription factor zDC (Zbtb46, Btbd4) defines the classical dendritic cell lineage. J Exp Med. 2012;209(6):1153-1165.

30. Raker VK, et al. Myeloid cell populations and fibrogenic parameters in bleomycin- and $\mathrm{HOCl}$-induced fibrosis [published online ahead of print June 16, 2016]. Exp Dermatol. doi:10.1111/exd.13124.

31. Zaba LC, Fuentes-Duculan J, Steinman RM, Krueger JG, Lowes MA. Normal human dermis contains distinct populations of CD11c+BDCA-1+ 
dendritic cells and CD163+FXIIIA+ macrophages. J Clin Invest. 2007;117(9):2517-2525.

32. Whitfield ML, et al. Systemic and cell type-specific gene expression patterns in scleroderma skin. Proc Natl Acad Sci U S A. 2003;100(21):12319-12324.

33. Gardner H, et al. Gene profiling of scleroderma skin reveals robust signatures of disease that are imperfectly reflected in the transcript profiles of explanted fibroblasts. Arthritis Rheum. 2006;54(6):1961-1973.

34. Hayakawa I, et al. Increased cutaneous T-cell-attracting chemokine levels in sera from patients with systemic sclerosis. Rheumatology (Oxford). 2005;44(7):873-878.

35. Andréasson K, Gustafsson R, Rydell-Törmänen K, Westergren-Thorsson G, Saxne T, Hesselstrand R. Limited impact of fibromodulin deficiency on the development of experimental skin fibrosis. Exp Dermatol. 2016;25(7):558-561.

36. Scheja A, et al. Von Willebrand factor propeptide as a marker of disease activity in systemic sclerosis (scleroderma). Arthritis Res. 2001;3(3):178-182.

37. Christmann RB, et al. Interferon and alternative activation of monocyte/macrophages in systemic sclerosis-associated pulmonary arterial hypertension. Arthritis Rheum. 2011;63(6):1718-1728.

38. Christmann RB, et al. Association of interferon- and transforming growth factor $\beta$-regulated genes and macrophage activation with systemic sclerosis-related progressive lung fibrosis. Arthritis Rheumatol. 2014;66(3):714-725.

39. Lakota K, et al. Levels of adiponectin, a marker for PPAR-gamma activity, correlate with skin fibrosis in systemic sclerosis: potential utility as biomarker?. Arthritis Res Ther. 2012;14(3):R102.

40. Bergmann C, Distler JH. Canonical Wnt signaling in systemic sclerosis. Lab Invest. 2016;96(2):151-155.

41. Milano A, et al. Molecular subsets in the gene expression signatures of scleroderma skin. PLoS One. 2008;3(7):e2696.

42. Königshoff M, et al. WNT1-inducible signaling protein-1 mediates pulmonary fibrosis in mice and is upregulated in humans with idiopathic pulmonary fibrosis. JClin Invest. 2009;119(4):772-787.

43. Nkyimbeng T, et al. Pivotal role of matrix metalloproteinase 13 in extracellular matrix turnover in idiopathic pulmonary fibrosis. PLoS One. 2013;8(9):e73279.

44. Brasaemle DL. Thematic review series: adipocyte biology. The perilipin family of structural lipid droplet proteins: stabilization of lipid droplets and control of lipolysis. J Lipid Res. 2007;48(12):2547-2559.

45. Lu TT, Browning JL. Role of the Lymphotoxin/ LIGHT System in the Development and Maintenance of Reticular Networks and Vasculature in Lymphoid Tissues. Front Immunol. 2014;5:47.

46. Edwards AD, Chaussabel D, Tomlinson S, Schulz O, Sher A, Reis e Sousa C. Relationships among murine $\mathrm{CD} 11 \mathrm{c}$ (high) dendritic cell subsets as revealed by baseline gene expression patterns. J Immunol. 2003;171(1):47-60.

47. Chin RK, et al. Lymphotoxin pathway directs thymic Aire expression. Nat Immunol. 2003;4(11):1121-1127.

48. Rennert PD, Browning JL, Mebius R, Mackay F, Hochman PS. Surface lymphotoxin alpha/ beta complex is required for the development of peripheral lymphoid organs. J Exp Med. 1996;184(5):1999-2006.

49. Chen MC, et al. The role of apoptosis signal-regulating kinase 1 in lymphotoxin- $\beta$ receptor-mediated cell death. J Biol Chem. 2003;278(18):16073-16081.

50. Greenblatt MB, et al. Interspecies comparison of human and murine scleroderma reveals IL-13 and CCL2 as disease subset-specific targets. Am J Pathol. 2012;180(3):1080-1094.

51. Sulzmaier FJ, Jean C, Schlaepfer DD. FAK in cancer: mechanistic findings and clinical applications. Nat Rev Cancer. 2014;14(9):598-610.

52. Petty JM, Lenox CC, Weiss DJ, Poynter ME, Suratt BT. Crosstalk between CXCR4/stromal derived factor- 1 and VLA-4/VCAM-1 pathways regulates neutrophil retention in the bone marrow. J Immunol. 2009;182(1):604-612.

53. Kvien TK. Annals of the Rheumatic Diseases: an update. Ann Rheum Dis. 2010;69(6):943-944.

54. Scuderi N, et al. Human adipose-derived stromal cells for cell-based therapies in the treatment of systemic sclerosis. Cell Transplant. 2013;22(5):779-795.

55. Yoshimura K, et al. Cell-assisted lipotransfer for facial lipoatrophy: efficacy of clinical use of adipose-derived stem cells. Dermatol Surg.
2008;34(9):1178-1185.

56. Krautler NJ, et al. Follicular dendritic cells emerge from ubiquitous perivascular precursors. Cell. 2012;150(1):194-206.

57. Fornaro M, et al. Fibronectin protects prostate cancer cells from tumor necrosis factor- $\alpha$ induced apoptosis via the AKT/survivin pathway. J Biol Chem. 2003;278(50):50402-50411.

58. Jiao J, et al. Dendritic cell regulation of carbon tetrachloride-induced murine liver fibrosis regression. Hepatology. 2012;55(1):244-255.

59. Ivanov S, et al. CCR7 and IRF4-dependent dendritic cells regulate lymphatic collecting vessel permeability. JClin Invest. 2016;126(4):1581-1591.

60. Rice LM, et al. Fresolimumab treatment decreases biomarkers and improves clinical symptoms in systemic sclerosis patients. J Clin Invest. 2015;125(7):2795-2807.

61. Reed NI, et al. The $\alpha \mathrm{v} \beta 1$ integrin plays a critical in vivo role in tissue fibrosis. Sci Transl Med. 2015;7(288):288ra79.

62. Barnes J, Mayes MD. Epidemiology of systemic sclerosis: incidence, prevalence, survival, risk factors, malignancy, and environmental triggers. Curr Opin Rheumatol. 2012;24(2):165-170.

63. Maria ATJ, et al. Antifibrotic, antioxidant, and immunomodulatory effects of mesenchymal stem cells in HOCl-induced systemic sclerosis. Arthritis Rheumatol. 2016;68(4):1013-1025.

64. Tyndall A. Mesenchymal stem cell treatments in rheumatology: a glass half full?. Nat Rev Rheumatol. 2014;10(2):117-124.

65. Koni PA, Sacca R, Lawton P, Browning JL, Ruddle NH, Flavell RA. Distinct roles in lymphoid organogenesis for lymphotoxins alpha and beta revealed in lymphotoxin $\beta$-deficient mice. Immunity. 1997;6(4):491-500.

66. Mohammad S, Patel RT, Bruno J, Panhwar MS, Wen J, McGraw TE. A naturally occurring GIP receptor variant undergoes enhanced agonistinduced desensitization, which impairs GIP control of adipose insulin sensitivity. Mol Cell Biol. 2014;34(19):3618-3629.

67. Han Y, Lefebvre V. L-Sox5 and Sox6 drive expression of the aggrecan gene in cartilage by securing binding of Sox 9 to a far-upstream enhancer. $M o l$ Cell Biol. 2008;28(16):4999-5013. 